\title{
Doppelte Providenz. Die Rezeption einer neuplatonischen Tradition bei Berthold von Moosburg
}

\author{
Theo Kobusch \\ Rheinische Friedrich-Wilhelms-Universität Bonn
}

In seiner Schrift Zum ewigen Frieden (1795) unterscheidet I. Kant zwischen der "gründenden Vorsehung“ (providentia conditrix) des Welturhebers, „sofern sie in den Anfang der Welt gelegt wird“, und der „waltenden Vorsehung“ (providentia gubernatrix), die diesen Anfang nach „allgemeinen Gesetzen der Zweckmäßigkeit“ erhalten soll.

Diese Unterscheidung einer doppelten Providenz hat eine lange Geschichte, der hier im Sinne einer Genealogie nachgegangen werden soll. In ihr spielt das Mittelalter eine herausragende Rolle, auf der Seite der Franziskaner - wie hier nur erwähnt werden kann - vor allem Alexander von Hales und die Summa Halensis, auf der Dominikanerseite vor allem Berthold von Moosburg und seine philosophischen Mitstreiter. Die folgenden Ausführungen haben zum Ziel, den mittelalterlichen Diskurs über das Thema der doppelten Providenz unserem Verständnis näher zu bringen und den neuplatonischen Ursprung dieser Idee offenzulegen.

\section{Natürliche und willensmäßige Providenz}

\subsection{Berthold von Moosburg}

Berthold von Moosburghat seinen monumentalen Kommentar zur Elementatio theologica des Proklos von vorneherein unter die Differenz der doppelten Providenz, d.h. der providentia naturalis und der providentia voluntaria gestellt. Die natürliche und die willensmäßige Providenz sind zwei Gesichtspunkte, unter denen das göttliche Gute je verschieden betrachtet wird. Nach der Sichtweise der natürlichen Providenz werden so die „theologischen Elemente“, d.h. vor allem die metaphysischen Prinzipien und primordialen Ursachen sichtbar. Deswegen ist die Koordinierung dieser „Elemente“ der eigentlich thematische Stoff dieses proklischen Werkes, so daß sich auch der Name des 
Werkes von selbst ergibt. ${ }^{1}$ Genauer gesagt handelt es sich bei den "theologischen Elementen“ um die Sätze über Gott. ${ }^{2}$ Diejenige Disziplin, die diese theologischen Sätze zum Gegenstand hat, heißt traditionell „Metaphysik“. Berthold lehnt diese aristotelische Bezeichnung (wie auch die „Erste Philosophie“) aufgrund ihrer engen naturphilosophischen Implikationen ab für das theologische Prinzipienwissen und damit auch für das Wissen von der natürlichen Providenz, von dem bei Proklos die Rede ist. Er zieht dafür die Begriffe des "supersapientialen Wissens“ oder der „divinalen Philosophie“ im Sinne Platons vor, die beide das „unvergleichbare Überragen“ über die aristotelische Metaphysik ausdrücken, die nur das Ens inquantum ens zum Gegenstand hat. ${ }^{3}$

Demgegenüber umfaßt die „Ordnung der willentlichen Providenz" nicht das Wesen- und Naturhafte, sondern - mit den Worten Alberts des Großen das Gnadenhafte bzw. das, was die göttlichen Gnadengaben partizipiert, das Hierarchische, Engel und Menschen, das mit freiem Willen Ausgestattete, Lohn und Strafe. Deswegen wird Dionysius der „Theologe“ der willensmäßigen Providenz genannt. ${ }^{4}$ Es ist das Reich des Willens, um das es hier geht, daher der Name der Ordnung. Auch es ist ein ordo divinus, auch hier geht es um das göttliche Gute, nämlich in partizipierter Form. ${ }^{5}$ Berthold kann deswegen beide Formen der Providenz auch als zwei Arten des "Hervorgangs“ (processus) begreifen, die natürliche als einen wesenhaften, die willentliche als einen partizipativen. ${ }^{6}$ Das Wissen von dieser Ordnung der willentlichen Providenz ist und heißt bei Berthold genau wie das Wissen um die natürliche Providenz: „Theologie“, aber jetzt ist die Offenbarungstheologie gemeint. Beide Theologien, die philosophische Theologie Platons und die Offenbarungstheologie - deren Wissenschaftscharakter Berthold im Praeambulum seines Kommentars darzustellen sich bemüht - haben eine ganz ähnliche Struktur: Wie die

1 Berthold von Moosburg, Expositio super Elementationem theologicam Procli. Expositio super Elementationem theologicam Procli. Propositiones 1-13, hg.v. M.R. Pagnoni-Sturlese, L. Sturlese (Hamburg: Meiner, 1984), Expositio tituli I, S.45, L.278-283: Ex praedictis apparet, quod bonum divinum secundum ordinem providentiae naturalis est subiectum huius libri, quod exprimitur per li: ELEMENTATIO THEOLOGICA. [...] Materialis quidem subiectiva, quia in ipso tractatur per elementorum coordinationem de bono divino secundum ordinem providentiae naturalis; unde dicitur elementatio non qualiscumque, sed theologica, ad differentiam elementationis physicae, quam etiam iste auctor dicitur edidisse.

2 Berthold von Moosburg, Expositio tituli K, S.47, L.365-366: Auctor tamen vocat eas elementa theologica, id est propositiones, in quibus est sermo de divinis sive de Deo.

3 Berthold von Moosburg, Expositio, Praeambulum C, S.65, L.422ff.; L, S.48, L.401ff.

4 Berthold von Moosburg, Expositio, 5B, S.116, L.104.

5 Berthold von Moosburg, Expositio, 10A, S.179, L.88-18o, L.112.

6 Berthold von Moosburg, Expositio super Elementationem theologicam Procli. Propositiones 136-159, hg.v. F. Retucci (Hamburg: Meiner, 2007), 146B, S.9o, L.19. 
philosophische Theologie auf allgemeinsten Prinzipien beruht, die nicht bewiesen und nicht gewußt sind, sondern nur "glaubend" hingenommen werden können - Berthold zählt zu ihnen neben dem Widerspruchsprinzip auch die Prinzipien der Vielheit und des Produktiven, die für die Stabilisierung der Existenz des Einen und des Guten notwendig sind -, so ist auch die Offenbarungstheologie auf nur geglaubte Prinzipien gegründet, eben auf die articulifidei Christianae. $^{7}$

Wie sich die beiden Theologien zueinander verhalten, ist nicht ganz einfach zu sagen, weil Berthold ihre Funktion nicht direkt gegenüber stellt. Wenn wir aber wissen könnten, wie sich die natürliche Providenz und die willensmäßige Providenz zueinander verhalten, dann würde auch das Verhältnis der beiden Theologien klar werden. Nun hat Berthold, wie weiter unten ausgeführt werden wird, die willensmäßige Providenz als eine „Ergänzung“, also als eine Vervollständigung eines Ganzen bezeichnet (s.u. S.340-342). Er hat damit einen Begriff ins Spiel gebracht, der als terminus technicus in den Erkenntnislehren seiner Zeit, besonders aber bei Heinrich von Gent und Dietrich von Freiberg, eine überragende Rolle spielt. Wird seine Bedeutung nicht erkannt, dann kann man dem Anspruch dieser Erkenntnislehren nicht gerecht werden. Das gilt nun auch hier:Wenn die willensmäßige Providenz eine „Ergänzung“ der natürlichen ist und wenn jener die Offenbarungstheologie, dieser aber die philosophische Theologie zugeordnet sein soll, dann muß die Offenbarungstheologie nach Berthold, aber auch nach Dietrich als eine „Ergänzung“ der philosophischen Theologie und damit der natürlichen Vernunft angesehen werden. ${ }^{8}$

7 Berthold von Moosburg, Expositio, Praeambulum, S.53, L.13-20: Circa stabilitionem anitatis seu existentiae unius utitur quodam principio complexo, isto videlicet, multitudinem esse, sicut et stabiliendo anitatem boni utitur alio principio, scilicet productivum esse. Ista duo principia, quibus totius istius philosophiae structura sicut propriis fundamentis innititur, auctor supponit [...] nullo modo vel intellecta vel scita [...] sed solum credita, sicut et theologia, quae est de bono divino secundum ordinem providentiae voluntariae, fundatur in principiis creditis, quae sunt articulifidei Christianae. Den Begriff der anitas, d.h. der "Obheit“, also der Existenz, den Berthold auch der quiditas, also der Essenz gegenüberstellt (Praeambulum C, S.68, L.556), dürfte Berthold von Avicenna oder Meister Eckhart kennengelernt haben.

8 Man kann F. Retucci nur zustimmen, wenn sie Bertholds Verdienste um die Stärkung der natürlichen Vernunft gegenüber Thomas und der übrigen Scholastik herausstellt. Allerdings darf das nicht zu der falschen Meinung führen, als komme die Offenbarungstheologie im Werk Bertholds gar nicht vor oder habe gar keine Funktion. Cf. „Magister Thomas Anglicus Minor. Eine neue Quelle der Expositio super Elementationem theologicam Procli Bertholds von Moosburg - das ungedruckte Sapientiale des Franziskaners Thomas von York“, in Berthold of Moosburg, Expositio - Prop. 136-159, s.XXIII-XXXIX. Ähnliches gilt auch im Hinblick auf Dietrichs Werk, wie schon De subiecto theologiae zeigt. Wenn L. Sturlese in Homo divinus (S.152) sagt, daß an keiner Stelle des Bertholdschen Werkes „von Sakramenten, Kirche, Offenbarung, guten Werken und Glaube die Rede“ sei, so ist man auf den ersten Blick geneigt, 
Und um es ganz klar auszudrücken: „Complementum“ und alle Ableitungen dieses Wortstammes bezeichnen nicht etwa, wie die in meinen beiden genannten Aufsätzen angeführten Belege zeigen (s.u. Anm.33), ein gegenseitiges Ergänzen, sondern eindeutig ein einseitiges: Die Offenbarungstheologie ist nach Dietrich und Berthold die Ergänzung der natürlichen Vernunft, nicht umgekehrt. Das bedeutet, daß die Offenbarungstheologie eine Hilfestellung innehat, nicht den Primat, aber auch nicht gleichrangig ist mit der supersapientalen Metaphysik, sondern ihr untergeordnet. Bei allem Respekt vor den Intellekttheorien der beiden Denker (Dietrich und Berthold) ist diese Umkehrung des Ancilla-Verhältnisses, diese intellektuelle Revolution, die eigentliche Errungenschaft ihrer Philosophie, das einmalig Große innerhalb der mittelalterlichen Philosophie. Die heutige Theologie, besonders die katholische kirchlich verfaßte, die im Sinne der providentia specialis sich auf partikuläre Prinzipien stützt, täte gut daran, sich (im Sinne Dietrichs und Bertholds) ihrer dienenden Funktion gegenüber der natürlichen, allgemeinen Vernunft, die auf universalen Prinzipien beruht, zu besinnen und auf den Primatsanspruch in Leben und Wissenschaft zu verzichten.

Beide Ordnungen, die der natürlichen und der willensmäßigen Providenz, sind göttliche Ordnungen, sie sind auch beide kausale Ordnungen, ${ }^{9}$ wenngleich die willensmäßige nur im übertragenen Sinne. Beide Ordnungen unterscheiden sich dadurch, daß - wie schon Albert der Große gesagt hatte - erstere eine Wesensordnung ist, die zweite aber eine durch Partizipation an den göttlichen Gnadengaben begründete. Die natürliche Providenz ist das Reich des Autarken, die willensmäßige dagegen das Reich des Hierarchischen. ${ }^{10}$

ihm unumwunden zuzustimmen, auf den zweiten nur noch zögerlich: Von „Offenbarung“ ist tatsächlich nicht die Rede, wohl aber von articuli fidei, von "guten Werken“ auch nicht, wohl aber von Lohn und Strafe (bes. im Zusammenhang mit der providentia voluntaria), vom Glauben auch nicht, aber immerhin weiß Berthold, von der „Wissenschaft“ (scientia) den „Glauben“ ( fides) abzuheben und zeigt darüber hinaus, daß er mit allen Wassern der zeitgenössischen Terminologie um "Gewißheit" und „Evidenz" gewaschen ist (cf. Berthold von Moosburg, Expositio, Praeambulum B, S.6o, L.247-264). Zur Unterscheidung von philosophischer Theologie und Offenbarungstheologie bei Berthold s. W. Goris, „Das Gute als Ersterkanntes bei Berthold von Moosburg", in W. Goris (Hg.), Die Metaphysik und das Gute. Aufsätze zu ihrem Verhältnis in Antike und Mittelalter. Jan A. Aertsen zu Ehren (Leuven: Peeters, 1999), S.143-145.

9 Berthold von Moosburg, Expositio super Elementationem theologicam Procli. Propositiones 14-34, hg.v. L. Sturlese, M.R. Pagnoni-Sturlese, B. Mojsisch (Hamburg: Meiner, 1986), 21A, S.77, L.47-49: Ordo etiam causalis, qui est secundum originem extra manantem, est duplex, quia aut secundum providentiam naturalem aut voluntariam secundum distinctionem Augustini VIII Super Genesim ad litteram.

10 Berthold von Moosburg, Expositio, 10A, S.179, L.88-18o, L.112, bes. S. 179, L.99-102: Ex praedictis apparet differentia inter antarkiam et hierarchiam, quia antarkia est ordo divinus 
Beide Ordnungen stellen auch Ganzheiten dar, die eine je verschiedene Formbestimmtheit haben. Die Ordnung der natürlichen Providenz ist ein Ganzes durch eine „wesensmäßige Verknüpfung oder Verbindung (...), der notwendig eine Formbestimmtheit durch den wesentlichen Einfluß eines auf ein anderes folgt" ${ }^{\prime \prime}{ }^{11}$ Es liegt in der natürlichen Providenz als einer Ordnung und Verbindung von wesentlichen Ursachen begründet, daß sie in eine dreifache Kraft oder Macht unterteilt wird: In die schöpferische, die später bei Kant als providentia conditrix wieder auftaucht, in die zusammenhaltende und in die (um das Heil) sorgende bzw. bewahrende. Berthold beruft sich für diese Unterteilung auf Johannes Damascenus. ${ }^{12}$ Auch Alexander von Hales hat sie in dieser Weise aufgenommen. ${ }^{13}$

Auch die Ordnung der willensmäßigen Providenz ist ein Ganzes, allerdings gemäß der Partizipation an einem höheren Licht. In dieser Ordnung gibt es die Weitergabe des Lichtes von Höherstehenden an die Niedrigeren, denn hier gibt es keine causa essentialis. Und mögen hier auch intellektuelle

secundum condiciones supra positas in descriptione emanans a Deo iuxta dispositionem providentiae naturalis; hierarchia vero est ordo divinus emanans a Deo iuxta dispositionem providentiae voluntariae.

11 Berthold von Moosburg, Expositio, 145B, S.83, L.47.

12 Berthold von Moosburg, Expositio, 141A, S.45, L.25-27: Verum, quia in naturali providentia est ordo et connexio causarum essentialium et substantialium, ideo subdividitur in conditivam, contentivam et provisivam. Berthold von Moosburg, Expositio, 141A, S.46, L.35-37: Etenim generatio nostra conditricis eius virtutis est, et permanentia contentivae eius est virtutis, et gubernatio et salus provisae eius est virtutis. Cf. Berthold von Moosburg, Expositio super Elementationem theologicam Procli. Propositiones 108-135, hg.v. F. Retucci (Hamburg, Meiner, 2011) 121H, S.108, L.110-112: Verum, quia omne, quod est ratio producendi, qua aliquid potest se ipsum exserere sua causalitate et fecunditate secundum triplicem providentiam, scilicet conditivam, contentivam et conservativam, est et dicitur virtus sive potentia, qua ipsum providens praehabet, praecomprehendit et praeobtinet omnia, quibus providet, necessarium est in omni divino per essentiam esse potentiam sive virtutem [...]. Die Dreiteilung scheint schon bei Proklos vorweggenommen zu sein. Cf. Proclus, Théologie platonicienne,

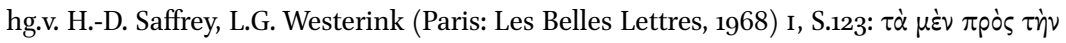

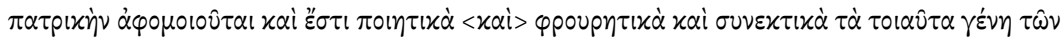

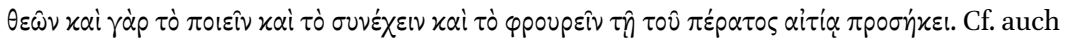
Proclus, Sur le Premier Alcibiade de Platon, I, hg.v. A.-Ph. Segonds (Paris: Les Belles Lettres,

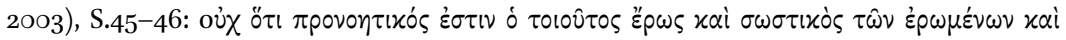

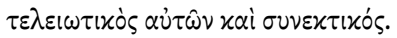

13 Alexander von Hales, Summa universis theologiae, ed. Quaracchi (Roma: Coll. S. Bonaventurae, 1924-1948), I, p. 1, inq. 1, tr. 5, s. 2, q. 3, tit. 1, c. 7, a. 1, n. 208, S.301: Providentia aliquando accipitur communiter, et sic pertinet ad virtutem conditivam et contentivam et provisivam: secundum hoc res ab ipsa sunt, permanent et gubernantur; et sic accipitur a Boethio, in libro Consolationis, cum determinat de providentia per comparationem ad fatum. 
Substanzen ihren Platz haben, so ist "dennoch keine von ihnen ein intellectus per essentiam ". ${ }^{14}$

Gegenstand der natürlichen Providenz Gottes ist die natürliche Ordnung der Ursachen, d.h. - im Sinne Eriugenas ausgedrückt - der primordialen Ursachen, also derer, die die Sorge Gottes „unmittelbar“ erfahren, während in der willensmäßigen Providenz die Sorge Gottes „auf eigene Weise“ sich vollzieht, nämlich durch Partizipation an der Güte Gottes. ${ }^{15}$ Die providentielle göttliche Sorge gilt also zuerst den „Göttern“, d.i. den übersubstantiellen Engeln. Berthold beruft sich in diesem Zusammenhang auf die Etymologie, sowohl auf die griechische wie die lateinische: Denn pronoia ist das vor dem Nous, und providentia meint die Tätigkeit des Provisors. ${ }^{16}$ Was die Etymologie des griechischen Ausdrucks angeht, so übernimmt er offenbar eine weitverbreitete neuplatonische Ansicht. ${ }^{17}$ Sie gibt der neuplatonischen Überzeugung Ausdruck, daß die Providenz die Sache Gottes ist, nicht des Geistes. ${ }^{18}$

14 Berthold von Moosburg, Expositio, 145B, S.83, L.47-58: [...] cum quidam sit totalis per essentialem colligationem seu connexionem, ordinationem ratione providentiae naturalis, quam necessario sequitur formalitas per essentialem influentiam unius in aliud [...] Est et alius ordo totalis in providentia voluntaria secundum participationem altioris luminis, [...] quam etiam exsequuntur taliter ordinata, inquantum superiora inferioribus communicant indita sibi lumina copiose. [...] cum nullum eorum sit causa essentialis. Licet enim sint intellectuales substantiae, nulla tamen earum est intellectus per essentiam [...].

15 Berthold von Moosburg, Expositio, 120E, S.10o, L.303-305: Secundum primum actum providentiae est ordo naturalis causarum, qui in hoc volumine exprimitur, quibus omnibus supereminent ipsae primordiales causae, quas auctor vocat deos. Berthold von Moosburg, Expositio, 120E, S.10o, L.321-323: Sicut ergo in providentia naturali Deus providet omnibus primordialibus causis immediate et per eas et in eis omnibus simpliciter, sic etiam in providentia voluntaria est suo modo.

16 Berthold von Moosburg, Expositio, 120 -prob., S.104, L.438-440: Igitur in diis est providentia prime. Ubi enim est pronoy, id est intellectus provisoris nisi in supersubstantialibus? Providentia vero est operatio provisoris, ut nomen insinuat.

17 Proclus, The Elements of Theology, hg.v. E.R. Dodds (Oxford: Clarendon Press, 19632 ${ }^{2}$, 120,

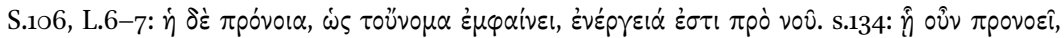

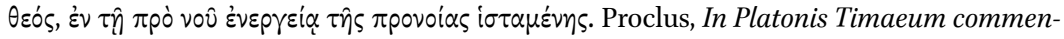
taria, hg.v. E. Diehl (Leipzig: Teubner, 1903, repr. Amsterdam: Hakkert, 1965), I, S.415: «ai

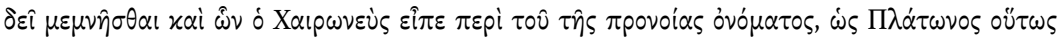

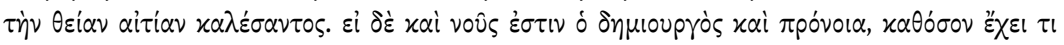

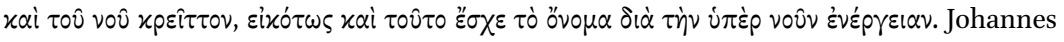
Philoponos, In Aristotelis De anima libros, hg.v. M. Hayduck (Berlin: Reimer, 1897), S.527:

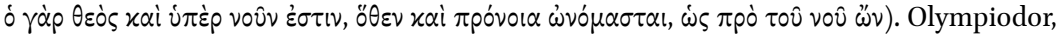
In Platonis Phaedonem Commentaria, hg.v. L.G. Westerink (Amsterdam: North-Holland, 1976), 13,2,15, S.167.

18 Proclus, In Platonis Parmenidem Commentaria, hg.v. C. Steel (Oxford: Oxford University

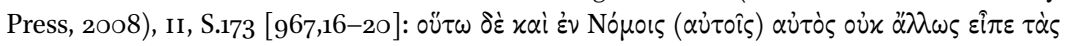

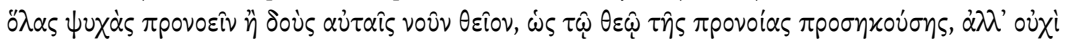

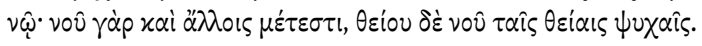


Mit Berufung auf Boethius versucht Berthold darüber hinaus den Unterschied beider Providenzarten auch als den zwischen der Providenz und dem Fatum deutlich zu machen. Die Providenz ist nach dem alles überragenden und bestimmenden neuplatonischen Schema jene Ordnung, die alles in eingefalteter Form enthält, was das Fatum als der Zeit unterworfene Ordnung entfaltet. ${ }^{19}$ Ob das Fatum durch gewisse göttliche dienende Geister oder die menschliche Seele oder die ganze Natur oder die Bewegung der Gestirne ausgeführt und die „fatale Reihe" des Geschehens gewebt wird, das will Berthold offenlassen, fest steht nur: Die Providenz ist die Ordnung des Einfachen, des Unbeweglichen, die Disposition des singulär und stabil zu Machenden, das Fatum dagegen die zeitliche und vielfältige Verwaltung desselben, die Ordnung des Beweglichen und des Raumzeitlichen. Deswegen kann man sagen: „Die Ordnung des Fatums geht aus der Einfachheit der Providenz hervor", ${ }^{20}$ d.h. das Fatum steht in den Diensten der göttlichen Providenz. Es bedeutet freilich auch: Alles, was dem Fatum unterworfen ist, ist auch der Providenz unterworfen, während der Kausalzusammenhang der wesentlichen Ursachen dem Fatum entzogen ist.

Ähnlichkeit und Differenz zwischen den beiden Providenzarten werden hier sichtbar: Die Providenz, gemeint ist die providentia naturalis, ist Gott „an sich“ (per se), das Fatum aber, das "Abbild“ von jener, ist nicht Gott, sondern nur „eine göttliche Sache“. ${ }^{21}$ Wir könnten das uns so übersetzen: Die „natürliche Providenz" ist das unmittelbare Handeln Gottes, das Fatum aber, die „willentliche Providenz", ist das vermittelte göttliche Handeln. Die göttliche Providenz ist "gewissermaßen“ ein "praktisches Wissen“, weil es der Wille Gottes ist, der sich auf alles erstreckt. ${ }^{22}$ Berthold hat in diesem Sinne das intellektuelle und das providentielle Erkennen als zwei Tätigkeiten eines substantiellen Intellekts unterschieden und so, wie das Mittelalter überhaupt, es vermieden, den göttlichen Intellekt „praktisch“ zu nennen. ${ }^{23}$

19 Berthold von Moosburg, Expositio, 141B, S.46, L.52-56: Providentia namque cuncta pariter, quamvis diversa, quamvis infinita complectitur, fatum vero singula dirigit in motu locis, formis ac temporibus distributa, ut haec temporalis ordinis explicatio in divinae mentis adunata prospectum providentia sit, eadem vero adunatio digesta atque explicata temporibus fatum vocetur. Zum complicatio-explicatio-Prinzip vgl. Th. Kobusch, „Complicatio und explicatio. Das ontologische Modell des Neuplatonismus“, in hg.v. G. Kapriev, The Dionysian Traditions, Turnhout, Brepols, 2021.

20 Berthold von Moosburg, Expositio, 141B, S.46, L.56-S.47, L.76.

21 Berthold von Moosburg, Expositio, 141B, S.48, L.103.

22 Berthold von Moosburg, Expositio, 120D, S.98, L.247: providentia tamquam practica scientia.

23 Berthold von Moosburg, Expositio, 134D-E, S.215, L.61-S.217, L.103 wird die Unterscheidung zwischen der Erkenntnis des Intellekts und der „Erkenntnis der Providenz" erwähnt. Daß der göttliche Intellekt „schlechthin spekulativ“ und nur per accidens praktisch ist, 


\subsection{Dietrich von Freiberg}

Neben Augustinus ist Dietrich von Freiberg der wichtigste Gewährsmann für die Lehre von der doppelten Providenz. Er hat sie zum Thema des Kapitels 20 seiner Schrift De animatione caeli gemacht. Dort kritisiert er die Position des Thomas von Aquin, der nicht genügend unterschieden habe die Ordungen der geistigen Substanzen einerseits und der himmlischen Körper, d.h. der Gestirne andererseits. Nach Dietrich haben sie aber von ihrer Natur gar keine Beziehung zueinander außer derjenigen, durch die sie beide aus dem ersten Prinzip, Gott, hervorgegangen sind, aber in je verschiedener Weise. Die geistigen Substanzen nämlich gehen in der Ordnung der willentlichen Providenz aus Gott hervor und sind hierarchisch und stufenmäßig unterschieden, und zwar nicht nur in ihrer Natur, sondern auch ihrem Willen und ihren hierarchischen Akten nach. Ihre Providenz gelangt bis zu uns. Die himmlischen Körper dagegen gehen in der Ordnung der natürlichen Providenz aus Gott hervor je unterschieden nach ihrer natürlichen Disposition und ihren natürlichen Eigentümlichkeiten und Bewegungen. Da aber beide Ordnungen, die der natürlichen Providenz und der willentlichen Providenz, „völlig disparat“ sind und das Eine mit dem Anderen nichts zu tun hat, können auch nicht die geistigen Substanzen, die zur Ordnung der willentlichen Providenz gehören, als Prinzipien der Himmelsbewegung, die selbst zur Ordnung der natürlichen Providenz gehört, gedacht werden. ${ }^{24}$

Doch nicht nur dieser Gedanke von der aus der willentlichen Providenz hervorgehenden hierarchischen Ordnung der geistigen Substanzen ist das historische Vorbild für Berthold. Was bei Berthold außerdem eindeutig auf Dietrich von Freiberg zurückzuführen ist, ist die Zuteilung der beiden Ordnungen zu den je verschiedenen Gegenstandsbereichen der philosophischen Theologie und der Offenbarungstheologie. Ihrer beider gemeinsamer Gegenstand ist zwar das "göttliche Seiende“ (ens divinum), aber ihre Betrachtungsweise ist je verschieden. Denn die philosophische Theologie, d.i. die Metaphysik oder Erste Philosophie betrachtet das, was wesenhaft göttlich ist und alles andere,

hat Heinrich von Gent in zwei Quaestionen ausführlich begründet: Heinrich von Gent, Summa (Quaestiones ordinariae), hg.v. G.A. Wilson, art. Xxxvı, qq. 4-5 (Leuven: Leuven University Press, 1994), S.105-130.

24 Dietrich von Freiberg, De animatione caeli, hg.v. L. Sturlese (Hamburg: Meiner, 1983), 20, S.3o, L.82-93: Huiusmodi autem substantiae spirituales omnino disparatae sunt in suis naturis et essentiis a substantiis corporum caelestium et nullum respectum et habitudinem secundum naturam habent ad ipsa nisi eam, qua ambo ista entium genera procedunt ab uno principio, Deo, sed tamen ordine diverso. Unum enim istorum, id est spirituum, [...] procedit in ordine voluntariae providentiae [...]. Aliud enim genus entium, scilicet corpora caelestia, procedunt a Deo in ordine naturalis providentiae [...]. 
insofern es auf dieses erste Prinzip hingeordnet ist. Die Metaphysik (scientia divina) betrachtet die Gesamtheit des Seienden, insofern sie aus Gott hervorgegangen ist, d.h. gemäß der Ordnung der natürlichen Providenz, in der die Dinge in ihrer Natur stehen und vom Ersten des Weltalls gemäß ihrer Modi und natürlichen Eigentümlichkeiten gelenkt werden, und über diese Ordnung der Natur hinaus achtet sie auf kein weiteres Ziel. ${ }^{25}$

"Unsere göttliche Wissenschaft der Heiligen aber", d.h. die Offenbarungstheologie, betrachtet das "göttliche Seiende" nach der Ordnung der willentlichen Providenz, d.h. die Dinge, insofern sie in die Ordnung der willentlichen Providenz gehören, wo es um Verdienst und Lohn und Strafe für ein gutes oder schlechtes Leben geht und die Möglichkeit, die ewige Glückseligkeit zu erlangen und überhaupt zu einem ferneren Ziel zu gelangen nach dem Ende dieser Welt - wenn die „göttliche Wissenschaft der Weisen dieser Welt vernichtet werden wird“. ${ }^{26}$

Vielleicht wurde Dietrich zu dieser Zuordnung der beiden Providenzarten zu den beiden Arten der Theologie von Heinrich von Gent angeregt, von dem er auch sonst beeinflußt ist. Dietrich hat ja in den siebziger Jahren in Paris studiert, und es ist sehr wahrscheinlich, daß er auch Heinrich von Gent gehört und kennen gelernt hat. Jedenfalls erinnert sich Dietrich rund zwanzig Jahre später, nämlich im 2. Buch von De intellectu et intelligibili, einer Disputation

25 Dietrich von Freiberg, De subiecto theologiae, 3 (5), hg.v. L. Sturlese (Hamburg: Meiner, 1983), S.281, L.69-75: Quia igitur in hac scientia tractatur de tota universitate entium - et secundum processum eorum a Deo [...] et haec sive secundum ordinem naturalis providentiae seu secundum ordinem voluntariae providentiae, secundum distinctionem Augustini VIII Super $(69 v b)$ Genesim - necesse est omnia convenire in una ratione subiecti, quod vocetur, sicut et vere est, ens divinum [...]. Dietrich of Freiberg, De subiecto theologiae 3 (8)-(9), S.281, L. 92 - S.282, L.104: Quamvis autem quantum ad considerationem primi philosophi talis etiam, quae dicta est, attributio entium ad primum principium attendatur, et propter hoc etiam potius dicitur apud philosophos scientia divina seu theologia, quam dicatur metaphysica - considerat enim primo et principaliter de ente divino, quod est divinum per essentiam [...] - nihilominus tamen nostra scientia, quam vere et simpliciter theologiam dicimus, distinguitur a scientia divina philosophorum. Scientia enim divina philosophorum considerat universitatem entium secundum ordinem providentiae naturalis, quo res stant in sui natura et secundum suos modos et proprietates naturales gubernantur per principem universitatis, nec ultra hunc naturae ordinem aliquem ulteriorem finem attendit.

26 Dietrich von Freiberg, De subiecto theologiae 3 (9)-(10), S.282, L.104-112: Nostra autem divina sanctorum scientia attenditur in entibus, secundum quod stant et disponuntur sub ordine voluntariae providentiae, in quo attenditur ratio meriti et praemii et ea, quae attenduntur circa bonam et sanctam vitam et adeptionem aeternae beatitudinis et perventionem ad finem ulteriorem sive in bono sive in malo etiam post terminum huius mundi, quando scientia divina sapientium huius mundi destruetur, I Cor. 13. Secundum praedicta igitur convenienter accipitur subiectum huius nostrae scientiae divinae, videlicet ut dicatur ens divinum secundum ordinem voluntariae providentiae. 
eines solemnis magister, und es spricht einiges dafür, daß Heinrich von Gent gemeint ist. ${ }^{27}$ Auf diese Weise zeigt sich ein weiteres Mal, daß Heinrich von Gent, dessen Einfluß in der Dietrich- und Eckhart-Forschung notorisch unterschätzt wird, ${ }^{28}$ die zentrale Figur in der zweiten Hälfte des 13. Jh. ist, die das Denken der Dominikaner wie auch und besonders der Franziskaner bestimmt hat. ${ }^{29}$

Was das hier verhandelte Problem angeht, so unterscheidet Heinrich im Anschluß an Augustinus die „allgemeine Providenz“ im Sinne der natürlichen und die "besondere Providenz" im Sinne der willentlichen. Die allgemeine Providenz bewegt zu jenen Erkenntnissen, die im Licht der natürlichen Vernunft gemacht werden können oder auch zu Handlungen, die aufgrund natürlicher Vernunft vollzogen werden. Die „spezielle“ Providenz dagegen bewegt zu solchen Erkenntnissen, die die Fähigkeit der natürlichen theoretischen Vernunft übersteigen und nur durch ein „übernatürlich“ eingeflößtes Licht möglich werden bzw. zu durch die göttliche Gnade ermöglichten Handlungen. ${ }^{30}$

27 Vgl. M. Führer, „Dietrich of Freiberg“, in E.N. Zalta (Hg.), The Stanford Encyclopedia of Philosophy (Fall 2020 Edition), URL = https://plato.stanford.edu/archives/fall2020/ente ries/dietrich-freiberg/ (29.11.2020).

28 In den sich als programmatisch gebenden „Einleitungen“ zu den kritischen Editionen Dietrichs und Bertholds von K. Flasch wird nicht einmal der Name Heinrichs einer Erwähnung für würdig gehalten. K. Flasch, „Einleitung“, in Dietrich von Freiberg, Opera omnia, vol. ı. Schriften zur Intellekttheorie, S.IX-XXVI. K. Flasch, "Einleitung", in Berthold of Moosburg, Expositio super Elementationem theologicam Procli. Prologus, Propositiones 1-13, S. xi-xxxviii.

29 Vgl. Th. Kobusch, Die Philosophie des Hoch- und Spätmittelalters (München: Beck, 2011), S.308-321.

30 Heinrich von Gent, Summa (Quaestiones ordinariae), hg.v. G.A. Wilson (Leuven: Leuven University Press, 2005), art. III, q. 5, S.263-264: Nunc autem sicut Deus agit et movet ut primus motor in movendo creaturas corporales motu corporali, sic agit et movet ut primus motor in movendo creaturas spirituales motu spirituali, et ideo sicut dupliciter movet, ut dictum est, creaturas corporales, una scilicet providentia generali et naturali, et altera providentia speciali et voluntaria, similiter congruit ut dupliciter moveat creaturam spiritualem. Motus autem eius est in intelligendo et volendo, et hoc scilicet providentia generali movendo eam lumine naturaliter sibi indito ad cognoscendum ea quae subsunt cognitioni naturali, et similiter ad ea agendum quae sibi ex naturalibus agere competit; providentia autem speciali movendo eam lumine supernaturaliter infuso ad cognoscendum quae sunt super facultatem rationis naturalis, et similiter voluntarie ad agendum quae sibi per gratiam agere competit, ut sic homo ordinetur, tam ex parte intellectus quam affectus [...]. Auch Thomas bestimmt die providentia specialis ganz im Sinne der providentia voluntaria als die besondere Sorge um die vernunftbegabten Wesen, cf. Summa contra Gentiles, III.111, hg.v. P. Marc, C. Pera, P. Caramello (Torino / Roma: Marietti, 1961); Summa Theologiae, I, 22,2, hg. Leonina (Roma: Ex Typ. Polyglotta S.C. de Propaganda Fide, 1888-1889). 
Zuletzt ist dies bemerkenswert, daß Dietrich die Willensordnung, also die Ordnung der Willensprovidenz, als die "Vervollständigung“ oder „Ergänzung“ der natürlichen Providenz versteht ${ }^{31}$. Berthold hat diese These Dietrichs zitiert. ${ }^{32}$ Sie ist insofern bemerkenswert, als dadurch ein Prinzip des Dietrichschen Denkens bestätigt wird. Dieses Prinzip besagt, daß die Tätigkeit des Intellekts, und das gilt sowohl für den theoretischen Intellekt bei der Konstituierung der ersten Intentionen wie auch für den praktischen Intellekt bei der Konstituierung z.B. des "Geldes“ oder des „Preises“ oder der positiven Gesetze, als eine „Ergänzung“ der Tätigkeit der Naturdinge anzusehen ist. ${ }^{33}$ Berthold hat dementsprechend auch die ersten und zweiten Intentionen unterschieden: Die ersten Intentionen sind das, was der Intellekt von der Sache als das washeitliche Sein aufgrund der formalen, d.h. in der Sache selbst liegenden Prinzipien konstituiert. Die ersten Intentionen heißen also so, weil sie zuerst von dem extramentalen Naturding angeregt und als solche vom Intellekt aufgenommen und quiditativ konstituiert werden. Die zweiten Intentionen dagegen, also so etwas wie Subjekt, Prädikat, Maior, Minor, Gattung, Species und andere Prädikabilien haben ihre Entität alleine durch den Intellekt. ${ }^{34}$ Berthold hat aber auch Dietrichs Lehre von der parallelen Struktur und Funktion des spekulativen und praktischen Intellekts aufgenommen, nach der z.B. der

31 Dietrich von Freiberg, De visione beatifica, hg.v. B. Mojsisch (Hamburg: Meiner, 1977), 4.3.2.(4), S.114, L.16-21: Et dico rationabile esse hoc et non dico necessarium esse, quia huiusmodi non fit ex necessitate ordinis, qui attenditur in providentia naturali, sed contingit ex sola Dei gratia et bonis meritis, quod pertinet ad ordinem voluntariae providentiae, qui est complementum et consummatio ordinis providentiae naturalis, quem duplicem ordinem in universo distinguit Augustinus VIII Super Genesim.

32 Berthold von Moosburg, Expositio, 129, S.182, L.299ff.

33 Cf. Th. Kobusch, „Begriff und Sache. Die Funktion des menschlichen Intellekts in der mittelalterlichen Philosophie“, in Internationale Zeitschrift für Philosophie 13/2(2004), 140157; Th. Kobusch, „Die Vernunftordnung als Vollendung der Naturordnung. Zur Funktion der menschlichen Vernunft in der mittelalterlichen Philosophie“, in T. Iremadze (mit H. Schneider, K.J. Schmidt) (Hgg.), Philosophy in Global Change: Jubilee volume dedicated to the 65th anniversary of Burkhard Mojsisch, Tbilisi: Nekeri, 2011, S.140-154.

34 Berthold von Moosburg, Expositio super Elementationem theologicam Procli. Propositiones 160-183, hg.v. U.R. Jeck, I.J. Tautz (Hamburg: Meiner, 2003), 177G, S.181, L.270-28o: Prima enim intentio est id, quod capit suam entitatem opere intellectus possibilis facti actu constituentis ipsum in esse quiditativo ex principiis formalibus, quae sunt partes definitionis; et dicitur res primae intentionis, quia est primo intentum a natura et sic respectum ab intellectu. Res vero secundae intentionis non sic capit suam entitatem, ut secundum se et primo sit aliquid extra intellectum, quo secundum se et primo sit respectum ab intellectu, sicut res primae intentionis, sed est forma quaedam habens se ex parte intellectus vel rationis, sub qua fit res rationabilis; et ideo quasi per accidens respicit ipsum intellectum, inquantum sub eo et per ipsum ratiocinatur de rebus, quas per se respicit. Est autem secunda intentio subiectum, praedicatum, maior vel minor extremitas, genus species et alia praedicabilia. 
„Preis“ als eine solche vom praktischen Intellekt dem Geld verliehene „komplementäre" Bestimmtheit anzusehen ist. ${ }^{35}$

Das Letztere zusammengefaßt:Wie der spekulative und praktische Intellekt durch seine jeweilige Tätigkeit die Tätigkeit des Naturdings "komplementär“ unterstützt und vollendet, so ist nach Dietrich und Berthold auch die „willentliche Providenz“ eine komplementäre Ergänzung der „natürlichen Providenz“.

\subsection{Augustinus}

Für die Unterscheidung der natürlichen und der willensmäßigen Providenz beruft sich Berthold sehr oft auf Augustinus, nämlich die klassische Stelle in De Genesi ad litteram, wo Augustinus die natürliche Providenz Gottes als die unvermittelte Erschaffung und verborgene Verwaltung der Naturwelt beschreibt, durch die er den Bäumen und Gräsern das Wachstum verleiht, aber auch das kosmische Geschehen regelt, die Elemente ins rechte Verhältnis zueinander bringt, und Geburt, Wachsen, Altern und Sterben der Lebewesen lenkt. Die willentliche Providenz Gottes aber ist die durch Menschen und Engel vermittelte Sorge um den Bestand der Zivilisation und Kultur. Sie ist daran zu erkennen, daß, gelehrt und gelernt' wird (doceri et disci), daß Felder bestellt, Staaten verwaltet, Künste geübt werden, und manch anderes, sei es in der himmlischen oder in der irdischen Gesellschaft getan wird und zwar so sehr, daß sogar die Schlechten, ohne daß sie es wüßten, zum Guten beitragen können. ${ }^{36}$ Die bedeutsame anthropologische Aussage Augustins an dieser

35 Berthold von Moosburg, Expositio, 32B, S.207, L.44-57. Im Hintergrund steht Heinrich von Gent, Summa (Quaestiones ordinariae) art. XLVII-LII, hg.v. M. Führer (Leuven: Leuven University Press, 2008), art. LI, q. 3, S.240. Vgl. Kobusch, „Die Vernunftordnung als Vollendung“. Auch Nikolaus von Straßburg, Summa, liber II, tract. 8-14, hg.v. T. Suarez-Nani (Hamburg: Meiner, 199o), tr. 9, q. 5, S.35-55 hat sich mit der Dietrichschen Lehre von den res primae intentionis und ihrer Verursachung durch den spekulativen und praktischen Intellekt kritisch auseinander gesetzt sowie auch mit Hervaeus Natalis' Kritik an Dietrich. Im Verlauf dieser Auseinandersetzung wird auch der Begriff des completum bzw. des complementum diskutiert (cf. Nikolaus von Straßburg, Summa, liber II, tr. 9, q. 5, S. 46, L.239-47, L.254).

36 Augustinus, De Genesi ad litteram, hg.v. J. Zycha (Prag / Leipzig / Wien: Tempsky / Freytag, 1894), VIII 9,17: atque in ipso quoque gemina operatio prouidentiae reperitur, partim naturalis, partim uoluntaria et naturalis quidem per occultam dei administrationem, qua etiam lignis et herbis dat incrementum, uoluntaria uero per angelorum opera et hominum; secundum illam primam caelestia superius ordinari inferius que terrestria, luminaria sidera que fulgere, diei noctis que uices agitari, aquis terram fundatam interlui atque circumlui, aerem altius superfundi, arbusta et animalia concipi et nasci, crescere, senescere, occidere et quidquid aliud in rebus interiore naturali que motu geritur; in hac autem altera signa dari, doceri et disci, agros coli, societates administrari, artes exerceri et quaeque alia siue in superna societate aguntur siue in hac terrena atque mortali, ita ut bonis consulatur et 
Stelle aber ist, daß diese doppelte Macht der Providenz - die Augustinus auch schon in früheren Schriften andeutet ${ }^{37}$ - auch im Menschen vorhanden ist, fast ganz parallel und bezogen sowohl auf den Leib als auch auf die Seele: die natürliche, d.h. dem Leib von innen gegebene Sorge äußert sich in den Bewegungen, durch die er entsteht, wächst und altert, die willentliche aber, indem er für den Lebensunterhalt, Kleidung und Pflege sorgen kann.

Die Unterscheidung zwischen dem Natürlichen und Willentlichen im Sinne einer Disjunktion ist eine Neuerung der christlichen Philosophie, eingeleitet durch Origenes und seine Anhänger. Diese Unterscheidung ist von eminenter Wichtigkeit für die weitere Geschichte der Philosophie. ${ }^{38}$ Denn sie bringt zu Bewußtsein, daß es hier um zwei irreduzible Welten geht, die jeweils eigene Gesetze haben: Die Welt der Natur mit ihren Naturgesetzen und die Welt des Moralischen, die den moralischen Gesetzen gehorcht. Später werden in diesem Sinne auch Natur und Freiheit unterschieden. Wenn Augustinus nun diese Unterscheidung auf die menschliche Form der Providenz, d.h. der Sorge anwendet, dann will er auf ein zweifaches Vermögen in unserer körperlichen und geistigen Existenz aufmerksam machen: Das natürliche Vermögen in der körperlichen Existenz ist die Bewegung des Entstehens, Wachsens und Alterns. Das moralische, d.h. in unsere Verantwortung gegebene, also unsere Freiheit betreffende dagegen ist die Ernährung, Kleidung und Körperpflege. Was die geistige Existenz angeht, so besteht das natürliche Element darin, daß die Seele als Lebensprinzip „lebt“ und Wahrnehmungen bzw. Empfindungen haben kann, während das „moralische“, von unserer Freiheit abhängige, im Lernen bzw. Erkennen und in der "Zustimmung“ besteht. Obwohl die Konzeption der doppelten göttlichen und der doppelten menschlichen Providenz ganz parallel strukturiert zu sein scheint, gibt es einen wichtigen Unterschied, den Augustinus wenig später auch ausdrücklich benennt: Die natürliche Providenz

per nescientes malos; in que ipso homine eandem geminam prouidentiae uigere potentiam: primo erga corpus naturalem, scilicet eo motu, quo fit, quo crescit, quo senescit; uoluntariam uero, quo illa ad uictum, tegumentum curationem que consulitur. similiter erga animam naturaliter agitur, ut uiuat, ut sentiat, uoluntarie uero, ut discat, ut consentiat. Cf. z.B. Berthold von Moosburg, Expositio, 141A, S.45, L.11ff.

Cf. Augustinus, De vera religione hg.v. J. Clémence, J. Pegon (Paris: Desclée de Brouwer, 1951), 22: [...] conditricem vero ac moderatricem temporum divinam providentiam [...].

38 S. Th. Kobusch, „Der Begriff des Willens in der christlichen Philosophie vor Augustinus“, in J. Müller, R. Hofmeister Pich (Hgg.), Wille und Handlung in der Philosophie der Kaiserzeit und Spätantike. Will and Action in Late Antique Philosophy (Berlin / New York: De Gruyter, 2010), S.277-30o. 
geschaffener Wesen kann keine Schöpfungsprovidenz im strengen Sinne der Schöpfung aus Nichts sein ${ }^{39}$.

Durch den Begriff der Zustimmung ist Augustinus zutiefst mit der stoischen Philosophie verbunden, in der die Zustimmung der eigentliche Hort der Freiheit darstellt, da sie im eigentlichen Sinne in unserer Macht steht $(\text { eph' hemin })^{40}$. Auch für Augustinus steht die Zustimmung im Zentrum der Freiheitslehre. Das ist schon daraus zu erkennen, daß nach Augustins durchgehender Lehre die Sündhaftigkeit der Sünde in der Zustimmung zum Bösen besteht ${ }^{41}$. Sie ist Sache des Willens ${ }^{42}$. Deswegen ist sie in De Genesi ad litteram VIII 9,17 der willentlichen Providenz zugeordnet.

Augustinus kommt in derselben Schrift - manchmal auch in anderen Schriften - noch mehrere Male auf die Lehre von der doppelten Providenz zurück und damit auch auf die Zweiteilung der gesamten Schöpfung in natürliche und willentliche Bewegungen. Daraus geht hervor, daß die göttliche Providenz eine zweifache Funktion hat: eine natürliche, durch die „Naturen“, d.h. die Wesenheiten zur Existenz gebracht werden, und eine „willentliche“, d.h. eine lenkende, durch die die verschiedenen Willen auf den richtigen moralischen Weg gelenkt und entsprechend ihrem Leben auch belohnt oder bestraft werden ${ }^{43}$. Damit sind wir bei der Vorstellung von der göttlichen Schöpfungs-

39 Augustinus, De Genesi ad litteram IX 15, S.288: quapropter cum deus uniuersam creaturam suam bipertito quodam modo opere prouidentiae, de quo in superiore libro locuti sumus, et in naturalibus et in uoluntariis motibus administret, creare naturam tam nullus angelus potest quam nec se ipsum.

40 Cf. Th. Kobusch, „Zustimmung I. Antike; Mittelalter“, in J. Ritter, K. Gründer, G. Gabriel (Hgg.), Historisches Wörterbuch der Philosophie (Basel / Stuttgart: Schwabe AG, 2005), S.1457-1465.

41 Cf. die berühmte Lehre von der Dreistufung der Sünde: suggestio, delectatio, consensio in De sermone Domini in monte I c.34.

42 Augustinus, De catechizandis rudibus, hg.v. I.B. Bauer (Turnhout: Brepols, 1969), 18: propria uoluntate consensit. Augustinus, Contra Secundinum, §14, hg.v. R. Jolivet, M. Jourjon (Paris: Desclée de Brouwer, 1961): dicis enim tunc animam uoluntate sua peccare, dum consentit malo. Augustinus, Epistulae, hg.v. K.D. Daur (Turnhout: Brepols, 2009), CVIII,7, S.71: Qui tetigerit pollutum, pollutus est, sed consensione uoluntatis.

43 Augustinus, De Genesi ad litteram viII 12,25: neque enim tale aliquid est homo, ut factus deserente eo, quifecit, possit aliquid agere bene tamquam ex se ipso; sed tota eius actio bona est ad eum conuerti, a quo factus est, et ab eo iustus, pius, sapiens beatus que semper fieri, non fieri et recedere, sicut a corporis medico sanari et abire, quia medicus corporis operarius fuit extrinsecus seruiens naturae intrinsecus operanti sub deo, qui operatur omnem salutem gemino illo opere prouidentiae, de quo supra locuti sumus. Augustinus, De Genesi ad litteram VIII 19,38: locus itaque magnus neque praetereundus proponitur, ut intueamur, quantum possumus, quantum ipse adiuuare et donare dignatur, opus diuinae prouidentiae bipertitum, quod superius, cum de agricultura loqueremur, transitoria quadam occasione perstrinximus, ut inde iam inciperet legentis animus hoc adsuescere contueri, quod adiuuat 
und Vergeltungsprovidenz angekommen, deren Ursprung in der Philosophie des Neuplatonismus liegt - wie Augustinus selbst zu wissen scheint (De civitate Dei, X 17).

Neuplatonismus: Schöpfungs- und Vergeltungsprovidenz

\subsection{Porphyrios und Jamblich}

Doch mögen Berthold und Dietrich und auch Augustinus noch so sehr der Lehre von der doppelten Providenz das Mäntelchen des Christlichen, d.h. der "christlichen Philosophie" umgehängt haben, indem sie die Konzeption des Willens damit verbunden haben, so ist der neuplatonische Ursprung dieser Lehre doch nicht zu übersehen.

Die göttliche Providenz war ein überragend wichtiges Thema für die antike, besonders auch die spätantike Philosophie und zwar sowohl für die pagane wie auch die christlichePhilosophie. Das bezeugen allein schon die zahlreichen Titel Peri pronoias, die wir kennen, so etwa von Chrysipp, Claudius Aelian, Philo von Alexandrien, Antiphon, Alexander von Aphrodisias, Clemens Alexandrinus, Johannes Chrysostomos, Theodoret, Proklos, Synesios, Hierokles, daneben

plurimum, ne quid indignum de ipsa dei substantia sentiamus. Augustinus, De Genesi ad litteram VII 23,44: ergo dei prouidentia regens atque administrans uniuersam creaturam, et naturas et uoluntates, naturas, ut sint, uoluntates autem, ut nec infructuosae bonae nec inpunitae malae sint [...]. Augustinus, De Genesi ad litteram VIII 24,45: et ideo deus bipertito prouidentiae suae opere praeest uniuersae creaturae suae, naturis, ut fiant, uoluntatibus autem, ut sine suo iussu uel permissu nihil faciant. Augustinus, De Genesi ad litteram VIII 26,48: sed in opere diuinae prouidentiae ista cognoscere non in illo opere, quo naturas creat, sed in illo, quo intrinsecus creatas etiam extrinsecus administrat [...]. Augustinus, De Genesi ad litteram IX 14,24: uomodo haec adduxerit deus ad adam, ne carnaliter sapiamus, adiuuare nos debet, quod de bipertito opere diuinae prouidentiae in libro superiore tractauimus. Augustinus, De Genesi ad litteram IX 15,28: quapropter cum deus uniuersam creaturam suam bipertito quodam modo opere prouidentiae, de quo in superiore libro locuti sumus, et in naturalibus et in uoluntariis motibus administret, creare naturam tam nullus angelus potest quam nec se ipsum. Augustinus, De Genesi ad litteram IX 18,33: habet ergo deus in se ipso absconditas quorundam factorum causas, quas rebus conditis non inseruit, eas que inplet non illo opere prouidentiae, quo naturas substituit, ut sint, sed illo, quo eas administrat, ut uoluerit, quas, ut uoluit, condidit. Cf. Augustinus, Enarrationes in Psalmos, hg.v. E. Deckers, I. Fraipont (Turnhout: Brepols, 1956), 145, 13: ad prouidentiam uniuersitatis, qua creauit omnia et mundum regit: homines et iumenta saluos facies, domine. Augustinus, De opere monachorum, hg.v. J. Zycha (Wien: Tempsky, 1900), 26,34, S.583: cum eius sapientissima prouidentia usque ad ista creanda et gubernanda perueniat. Augustinus, De civitate Dei, hg.v. B. Dombart, A. Kalb, G. Bardy, G. Combès (Paris: Institut d'études augustiniennes, 2014), I, 36: et quod Deus uerus mundum condiderit et de prouidentia eius, qua uniuersum quod condidit regit. 
wichtige Kapitel mit diesem Titel in Schriften wie z.B. in Epiktet I 6, Nemesius De natura hominis c.42, das Carmen Peri pronoias von Gregor von Nazianz oder Kap. 17 in De deis et mundo von Sallust oder schließlich das entsprechende Kapitel in der Expositio fidei des Johannes Damascenus. Dem entsprechen ebensoviele Titel auf der lateinischen Seite von Philo von Alexandrien, Seneca, Augustins q. 27 von De div. quaest., sein Sermo De providentia Dei u.a.m.

Die Lehre von der doppelten göttlichen Providenz, d.h der Schöpfungs- und der Vergeltungsprovidenz, hat im Rahmen dieses allgemeinen Interesses an der Providenzthematik einen besonderen Stellenwert. Ihre eigentliche Heimat ist der Neuplatonismus. Doch von welchem Autor könnte sie stammen? Bei Plotin z.B., von dem wir ja zwei Providenzschriften haben, finden wir sie nicht, wohl aber bei fast allen späteren Neuplatonikern. W. Theiler hat in einem großartigen Aufsatz erstmals das Bewußtsein der modernen Forschung für dieses Thema geweckt ${ }^{44}$. Mit der Aufdeckung dieses spezifisch neuplatonischen Grundsatzes hat Theiler jedoch eine These verbunden, die gewagt zu sein scheint. Die These besagt, daß aus der auffälligen Übereinstimmung zwischen dem Christen Origenes und dem Neuplatoniker Hierokles das „System des Ammonios Sakkas" ablesbar sei. DieThese ist gewagt, weil sie in der Hauptsache nicht greift - wie Theiler auch selbst weiß (p. 30). Denn nach Origenes ist die geschaffene geistige Welt ontologisch differenz- und grenzenlos - erst der geschaffene Wille macht die Wesensgrenzen -, nach Hierokles aber gibt es „demiurgische“, d.h. von Gott gesetzte ontologische Grenzen ${ }^{45}$. Doch auch wenn man so gegenüber diesem Versuch der Konstruktion des ammonischen Systems skeptisch sein muß, bleibt das unvergeßliche Verdienst dieses Aufsatzes, in der Forschung wegweisend auf die Bedeutung der neuplatonischen Lehre von der Schöpfungs- und Vergeltungsprovidenz hingewiesen zu haben.

Wir können diese Lehre erstmals deutlich bei Porphyrios finden. Der Christengegner ist in manchem der christlichen Lehre verblüffend nah. Das gilt nicht nur etwa für die Lehre von der Schöpfung, sondern auch für die hier ins Auge gefaßte Providenzlehre. In seiner christentumsnahen Schrift Ad Marcellam ist der Providenzglaube von entscheidender Bedeutung. Die einen, so sagt Porphyrios, haben sich „durch Erkenntnis und festen Glauben“ die Überzeugung erworben, daß Gott ist und alles verwaltet, d.h. daß er die Welt erschaffen hat und alles providentiell regelt, indem mit Hilfe göttlicher Engel

44 W. Theiler, „Ammonios der Lehrer des Origenes“, in Id., Forschungen zum Neuplatonismus (Berlin: De Gruyter, 1966), S.1-45. Zur Schöpfungs- und Vergeltungsprovidenz: S.9. 13. 15 f. 42. 44.

S. Th. Kobusch, „Origenes und Pico. Picos Oratio im Licht der spätantiken Philosophie“, in A. Fürst, Chr. Hengstermann (Hgg.), Origenes Humanista. Pico della Mirandolas Traktat De salute Origenis disputatio (Münster: Aschendorff, 2015), S.141-159. 
bzw. guter Dämonen, denen nichts entgeht, die Handlungen der Menschen beobachtet werden. Auf diese Weise lernen solche Gottgläubigen einerseits durch ihr sittliches Leben die Götter kennen, andererseits werden sie auch durch die erkannten Götter erkannt ${ }^{46}$. Die anderen dagegen - sagt Porphyrios -, die weder an Gottes Existenz glauben noch an die providentielle Verwaltung des Universums durch Gott, haben die "Strafe des Rechts" erhalten, indem sie weder sich selbst noch anderen darin vertrauten, daß Götter existieren und daß das Universum nicht durch irrationalen Zufall verwaltet wird. Diese Ungläubigen haben sich selbst in die größte Gefahr begeben und sich ihrerseits einem irrationalen Impuls ergeben und alles getan, was nicht recht ist, indem sie versuchten, den Glauben an die Götter zu zerstören. Im Gegensatz zu den ersteren haben sie ein schlechtes Leben gewählt, wissen nichts von den Göttern, werden aber von den Göttern und dem göttlichen Recht erkannt. Selbst wenn sie glauben sollten, die Götter zu verehren und von der Existenz der Götter überzeugt zu sein, dabei aber das Moralische vernachlässigen, dann handelt es sich in Wirklichkeit um eine Leugnung der Götter ${ }^{47}$. Denn weder ein irrationaler Glaube noch eine blinde Verehrung würden ohne ein sittliches Leben eine Annäherung an Gott sein. Mit dem „irrationalen Glauben“ zielt Porphyrios kritisch auf eine fideistische Gruppe der frühen Christen ${ }^{48}$. Da die Ausgangspunkte für alle so unterschiedlich sind, ist es am gerechtesten anzunehmen, daß für Gott die Reinheit der Gesinnung zählt, da diese jeder aufgrund seines freien Willens erreichen kann ${ }^{49}$.

Auch sonst spielt die Doppelung von Gottes Existenz und Providenz, die später als natürliche und willensmäßige Providenz üblich wird, bei Porphyrios eine wichtige Rolle. Ihre Leugnung nennt er die „erste“ und „zweite Gottlosigkeit“50. Bei Jamblich stehen die Schöpfung und Providenz der Götter

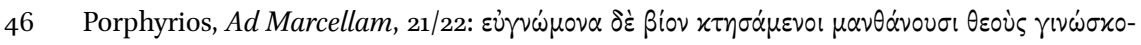

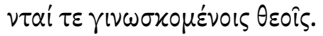

47 Porphyrios, Ad Marcellam, hg.v. A. Nauck und übers. von W. Pötscher (Leiden: Brill, 1969),

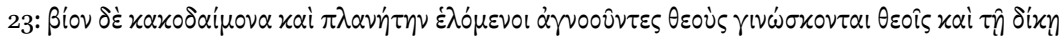

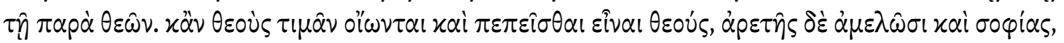

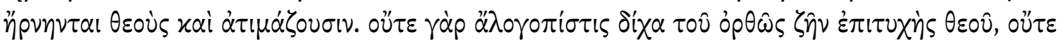

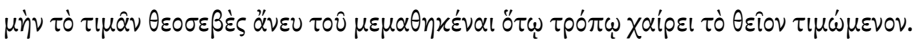

48 Cf. Th. Kobusch, Christliche Philosophie. Die Entdeckung der Subjektivität (Darmstadt: Wissenschaft Buchgesellschaft 2006), S.98.

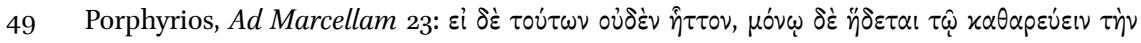

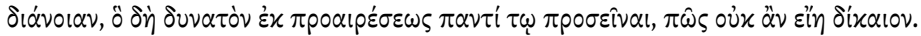

50 Porphyrios, In Platonis Timaeum Commentariorum Fragmenta, hg.v. A.R. Sodano (Neapel:

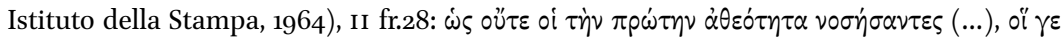

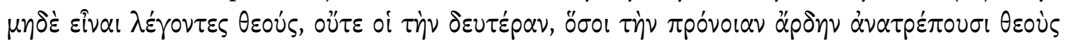

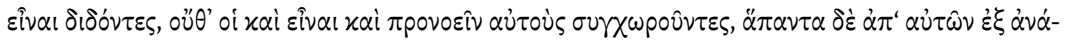

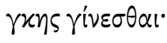


ganz nah beieinander ${ }^{51}$. Die Atheisten verraten eine einfache Denkweise: Weil sie die Art und Weise der Schöpfung und providentiellen Sorge Gottes nicht durchschauen, leugnen sie beides strikt $\mathrm{ab}^{52}$. Die Providenz wird dabei als Vergeltungsprovidenz verstanden. In diesem Sinne ist auch das Schlechte providenzgemäß $\Re^{53}$.

\subsection{Die späteren Neuplatoniker}

Die Lehre von der doppelten Providenz wird im Neuplatonismus auf vielfache Art und Weise zum Ausdruck gebracht. Proklos hat das Verhältnis der Schöpfungsprovidenz und der Vergeltungsprovidenz, (d.h. in bezug auf das Letztere: was bei Berthold von Moosburg „Fatum“ genannt wird), in klassischer Weise bestimmt: Die Heimarmene und damit das Recht hängt von der göttlichen Schöpfungsprovidenz ab und folgt den Bestimmungen des Schöpfers, indem es das göttliche Gesetz durchsetzt ${ }^{54}$. Götter, Dämonen und Seelen helfen bei diesem Werk der Gerechtigkeit ${ }^{55}$. Wie Philoponos und Johannes Damascenus übereinstimmend überliefern, ist es das Verdienst der proklischen Providenzschrift, gezeigt zu haben, daß die schöpferische und providentielle Macht Gottes bis zu den äußersten und feinsten Enden der Schöpfung reicht ${ }^{56}$.

Proklos hat diese Zweiheit auch als zwei kosmische Umdrehungen beschrieben, die an die oben erwähnte augustinische Zweiteilung der Providenz erinnert: Die eine ist rein intellektuell und hat eine für die Seelen anagogische

$51 \quad$ Jamblich, De mysteriis, III 16: "H

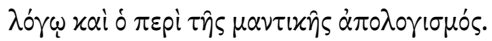

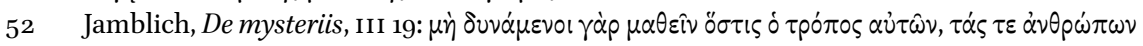

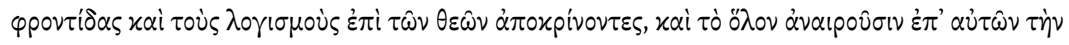

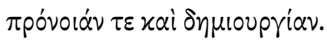

53 Jamblich, Theologumena arithmeticae, hg.v. V. de Falco (Leipzig: Teubner, 1922), S.45: $\tau \dot{\alpha}$

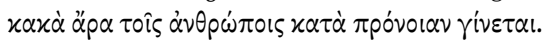

54 Proclus, In Platonis Rempublicam Commentaria, hg.v. W. Kroll (Leipzig: Teubner, 19o1, repr.

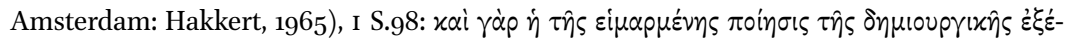

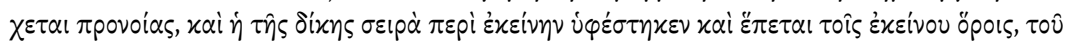

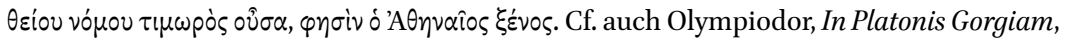

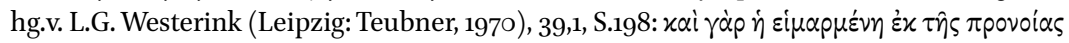

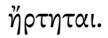

55 Proclus, In Platonis Rempublicam Commentaria, II S.135.

56 Johannes Philoponos, De aeternitate mundicontra Proclum, hg.v. H. Rabe(Leipzig:Teubner,

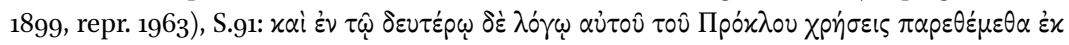

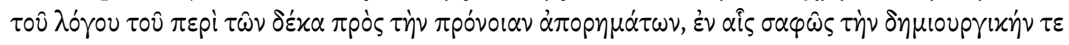

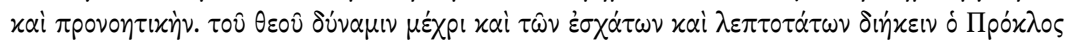

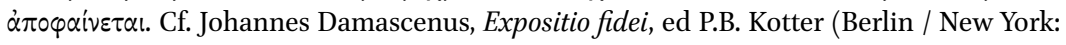
De Gruyter, 1973), S.42. 
Funktion, die andere führt im Gegensatz dazu in die Welt der Natur hinein, die eine ist unsichtbar und durch die göttliche Providenz gelenkt, die andere ist sichtbar und stellt die Entfaltung der natürlichen Providenz in der Ordnung der Heimarmene dar ${ }^{57}$.

Die Unterscheidung zwischen dem Sichtbaren und dem Unsichtbaren gilt auch im Hinblick auf den Menschen selbst: Die physischen Eigenschaften wie Schönheit oder Größe der Gestalt sind Geschenke der Heimarmene oder der Natur, die das Seelenheil und eine höhere Lebensart bewirkenden Kräfte dagegen sind die Keimkräfte der göttlichen Providenz ${ }^{58}$. So ergibt sich ganz allgemein: Die Heimarmene ist die für die Welt des sinnlich Wahrnehmbaren zuständige Providenz, die von der unsichtbaren der Götter abhängig ist ${ }^{59}$. Die Heimarmene ist dabei auch der Inbegriff für die in der göttlichen Schöpfungsprovidenz präexistierenden notwendigen Gesetze der Natur und des Sittlichen, die als Naturgesetze und Sittengesetze den einzelnen Seelen apriori eingepflanzt werden ${ }^{60}$.

Wiederum anders und sehr aufschlußreich auch im Hinblick auf die Lehre vom Einen ist die Bemerkung Proklos', daß die eine Providenz des Göttlichen ganz transzendent gegenüber dem von ihr Umsorgten ist, während die andere den Charakter des „Koordinierten“ hat ${ }^{61}$. Diese Lehre hat ihre Entsprechung, oder sollten wir sagen: ihren Grund in der Ontologie: Im Parmenideskommentar hat Proklos in Auseinandersetzung mit der Theorie des historischen Parmenides das absolute Eine als das "Transzendente“ (exhairemenon), d.h als das Zusammenhanglose definiert, dem das „Hen on“

57 Proclus, Théologie platonicienne, hg.v. H.D. Saffrey, L.G. Westerink (Paris: Les Belles Lettres,

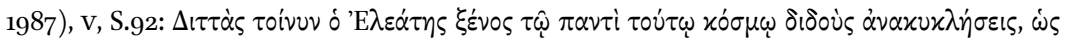

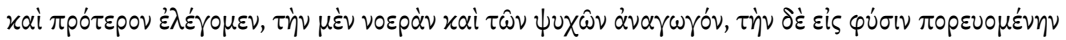

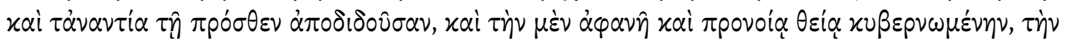

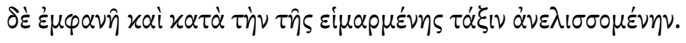

$5^{8}$ Proclus, Sur le Premier Alcibiade, 134,1-14, I, S.111.

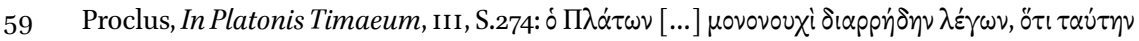

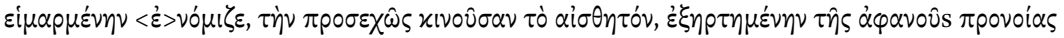
$\tau \hat{\omega} v \theta \varepsilon \hat{\omega} v \cdot$ Cf. Olympiodor, In Platonis Gorgiam, c.39,1.

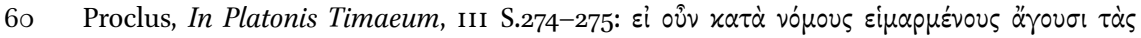

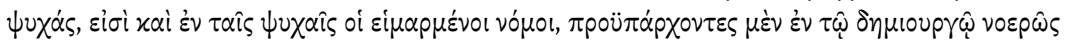

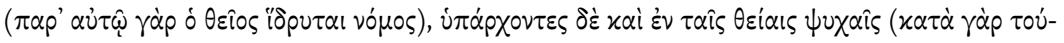

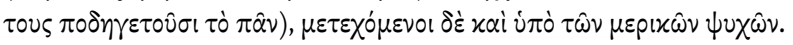

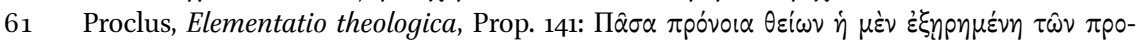

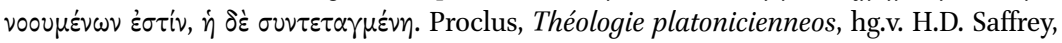
L.G. Westerink (Paris: Les Belles Lettres, 1997), vi, S.77: $\Delta \imath \tau \tau \dot{\eta} \nu ~ \gamma \alpha \dot{\alpha} \rho$ xà

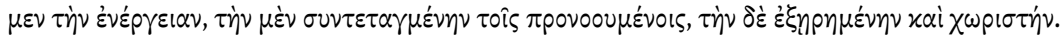


als das „Koordinierte“, d.h. als das mit dem Vielen irgendwie Verbundene gegenübersteht ${ }^{62}$.

Nach Hermias, dem Zeitgenossen des Proklos, sind die Schöpfungsprovidenz und die Vergeltungsprovidenz zwei unterschiedliche Aspekte desselben: Die Schöpfungsprovidenz hat den Charakter des Existenzstiftenden (hypostatike), die Vergeltungsprovidenz dagegen stiftet das „Heil“ (sostike). Mit dem Letzteren knüpft Hermias an Porphyrios' Vorstellung vom universalen Weg zum Heil der Seele $a^{63}$.

Am deutlichsten hat Hierokles von Alexandrien die These von der doppelten Providenz Gottes vertreten. In seiner Schrift Über die Providenz, von der wir Teile bei Photius überliefert haben, knüpft er an Platons Lehre von Gott als dem Schöpfer und Vater aller Dinge an. Seine Königsherrschaft besteht in seiner Providenz, die jeder Gattung das ihr Zukommende zuteilt. Das dieser Verfügung aber folgende Recht wird Heimarmene genannt. Sie ist die Providenz im Sinne der Vergeltung, die auf die freien und selbstmächtigen Handlungen der Menschen reagiert, d.h. sie korrigiert ${ }^{64}$. Die Vergeltung aber wird göttlicherseits in die Hände ätherischer Wesen, also der Engel gelegt, die von Gott als Richter eingesetzt werden und die Sorge um uns naturgemäß übernehmen ${ }^{65}$. Da, wie Hierokles bemerkenswerterweise sagt, die providentielle Beurteilung, die mit Recht und Gesetz die menschlichen Dinge ordnet, unserer Freiheit bedarf, die Heimarmene aber die Antwort auf unsere freien Handlungen ist, muß die Heimarmene, d.h. das rechtsprechende Verhältnis zu den menschlichen Seelen als ein Teil der göttlichen Providenz angesehen werden ${ }^{66}$.

62 Proclus, In Platonis Parmenidem, 714,5 [711,21].

63 Hermias, In Platonis Phaedrum Scholia, hg.v. C.M. Lucarini, C. Moreschini (Berlin: De

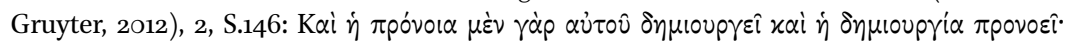

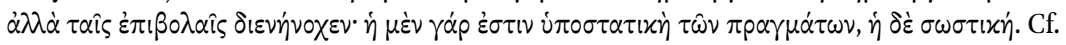

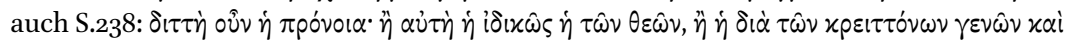

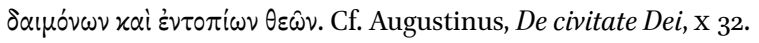

64 Hierokles, Peri Pronoias (bei Photius, Bibliotheka, hg.v. R. Henry (Paris: Les Belles Lettres,

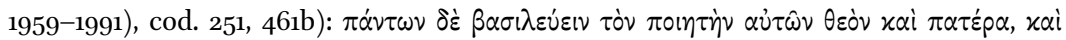

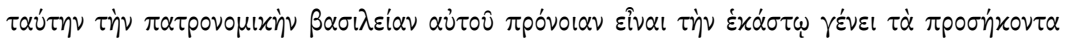

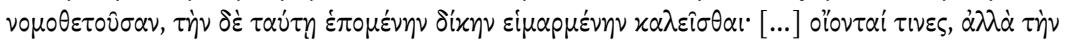

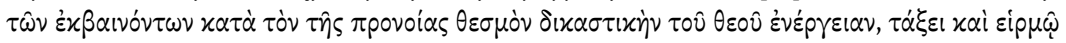

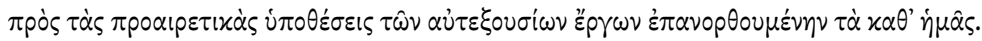

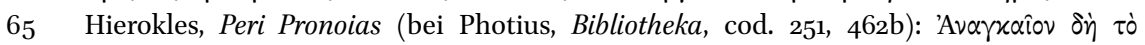

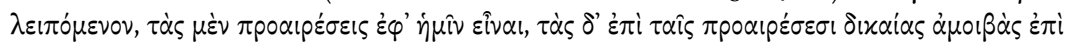

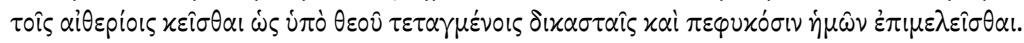

66 Hierokles, Peri Pronoias (bei Photius, Bibliotheka, cod. 251, 462b): xai

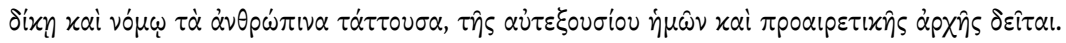


Was - wenn man so sagen kann - den Zuständigkeitsbereich der göttlichen Providenz angeht, so ergibt sich ein auffälliger Unterschied zur Lehre des Proklos, mit dem Hierokles sonst Vieles gemeinsam hat: „Die Tiere und Pflanzen sind der göttlichen Vorsehung entzogen“, hier herrscht der Zufall (hos tyche), sagt Hierokles ${ }^{67}$, während nach Proklos die Kompetenz der göttlichen Providenz bis zu den äußersten Enden des Universums und das bedeutet: bis zur Pflanzenwelt und toten Körperwelt reicht ${ }^{68}$.

Die göttliche Providenz im Sinne der providentia gubernatrix ist ihrem weitesten Begriff nach die Lenkung des Universums. Sie ist in diesem Sinne Gegenstand der Ersten Philosophie, d.h. der Metaphysik ${ }^{69}$. Es ist der „Theologe“, d.h. der Metaphysiker, der die Frage erörtert, ob alles aus Notwendigkeit geschieht oder auch aus Zufall, und wenn alles notwendigerweise geschieht, ob dann die Gründe für unsere Fehler in der Heimarmene, d.h. der providentia gubernatrix liegen oder, wenn doch Einiges in unserer Macht liegt im Sinne des eph 'hemin, ob wir dann nicht als autonome Wesen Sorge zu tragen haben für unsere Ausbildung und Moralität ${ }^{70}$. Nur unter dieser Voraussetzung der Freiheit des Menschen kann die lenkende Providenz auch als Straf- oder Vergeltungsprovidenz gedacht werden, die die Taten der Menschen nach ihrer

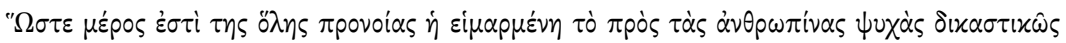

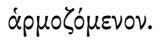

67 W. Theiler, „Ammonios der Lehrer des Origenes“, nf. Cf. Hierokles, In Aureum Pythagoreorum Carmen Commentarius, hg.v. F.W. Koehler (Stuttgart: Teubner, 1974), XI 28, S.51. Cf. Hierokles, Peri Pronoias (bei Photius, Bibliotheka, cod. 251, 466 a).

68 Proclus, De decem dubitationibus circa providentiam, hg.v. H. Boese (Berlin: De Gruyter,

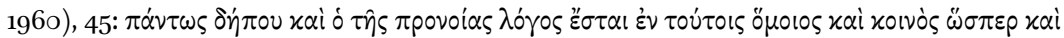

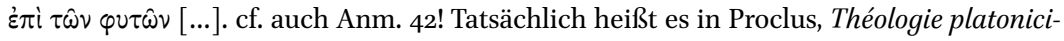

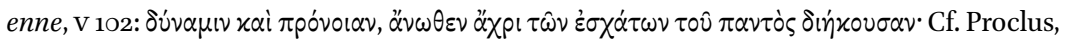
Sur le Premier Alcibiade, I 53,21, S.44.

69 Cf. Ammonios, In Aristotelis librum De interpretatione commentarius, hg.v. A. Busse

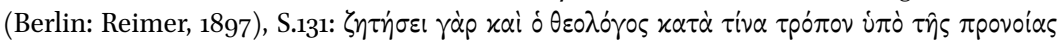

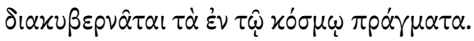

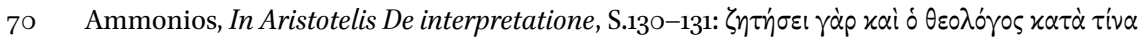

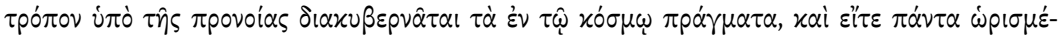

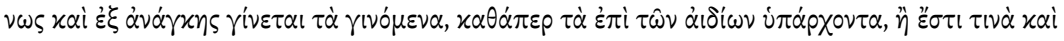

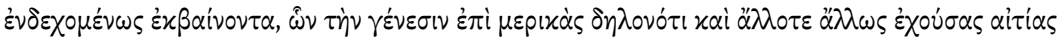

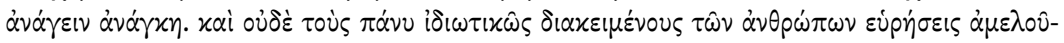

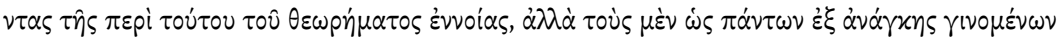

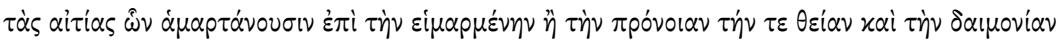

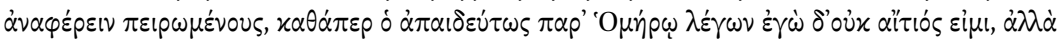

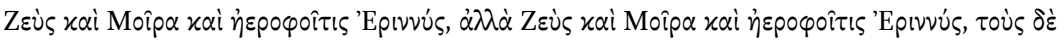

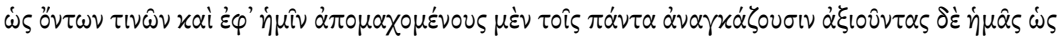

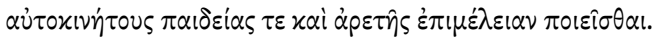


Würdigkeit vergilt ${ }^{71}$. Von dieser Vergeltungsprovidenz scheint der gesamte spätere Neuplatonismus zu sprechen ${ }^{72}$.

Nach Philoponos kümmert sich die göttliche Providenz nicht nur um unser Sein, d.h. um unsere Existenz im Sinne der providentia conditrix oder naturalis, sondern auch um unser Gutsein im Sinne der providentia voluntaria, die auch die ins Widernatürliche abgeglittene Seele nicht vernachlässigt, sondern ihrer Sorge teilhaftig werden läßt, indem sie ihr die Gelegenheit zur Selbstreinigung gibt ${ }^{73}$.

Die Schöpfungsprovidenz (demiurgike pronoia) definiert Simplikios in diesem Zusammenhang als die mit der göttlichen Güte koexistierende schöpferische Tätigkeit, durch die der Demiurg dem Universum die Ordnung verschafft hat ${ }^{74}$.

Wie Simplikios berichtet, ist der Gedanke einer lenkenden Providenz auf die alten Mythenerzähler zurückzuführen, nach denen das Meer ein Symbol der Entstehung der Seelen war. Das Schiff, das die Seelen zur Entstehung brachte, steht dabei für das zugeteilte Los (Moira) oder das unentrinnbare Schicksal (Heimarmene) oder wie man es sonst nennen soll. Der Lenker des Schiffes aber ist Gott, der das All lenkt und durch seine Providenz die Entstehung der Seelen und die entsprechende Vergeltung regelt ${ }^{75}$.

71 Cf. Ammonios, In Aristotelis Categorias commentarius, hg.v. A. Busse (Berlin: Reimer,

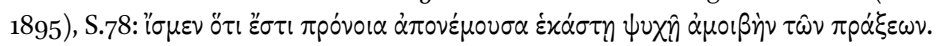

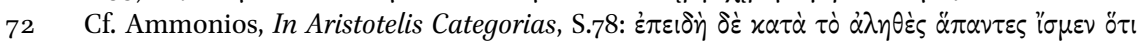

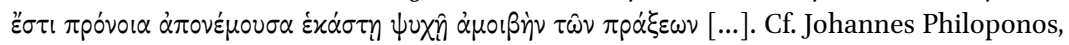
In Aristotelis Categorias commentarius, hg.v. A. Busse (Berlin: Reimer, 1898), S.127: xai

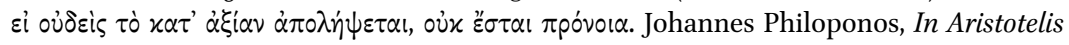

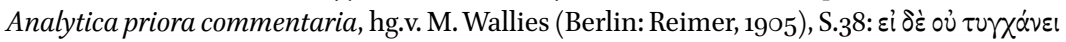

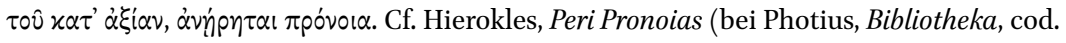

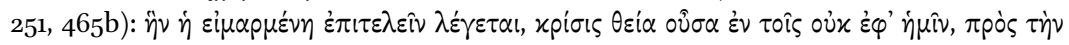

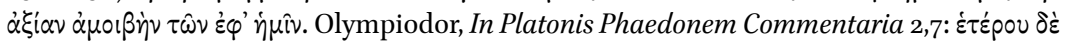

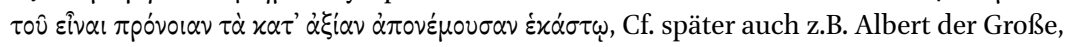
In IV Sententiarum, hg.v. A. Borgnet (Paris: Vivès, 1894), d. 43, a.1, S.498: Primum est de justitia et providentia, quae ordinat quamlibet naturam secundum gradum suae dignitatis.

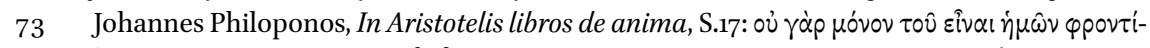

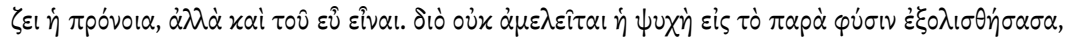

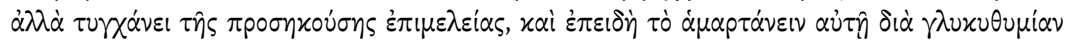

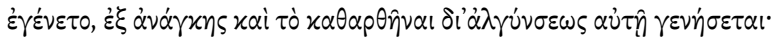

74 Simplikios, In Aristotelis Physicorum libros commentaria, hg.v. H. Diels (Berlin: Reimer,

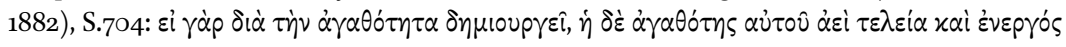

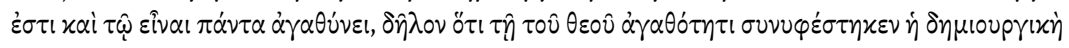

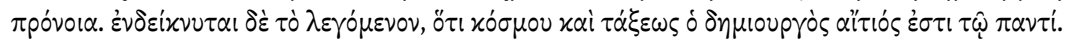

75 Simplikios, Commentarius in Epicteti enchiridion, hg.v. I. Hadot (Leiden / New York:

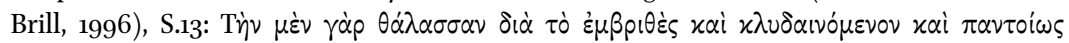

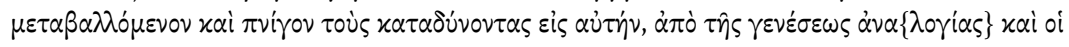


Wie Johannes von Damaskus später sicher ganz in neuplatonischem Sinne sagt, ist die Providenz im allgemeinen Sinne nichts Anderes als die Sorge Gottes um das Seiende ${ }^{76}$. Doch gerade bei diesem Begriff der Sorge kann der Unterschied zwischen der christlichen und nichtchristlichen Konzeption der göttlichen Providenz deutlich werden. Bei Simplikios nämlich treffen beide Konzeptionen aufeinander.

Auch wenn die Providenz als Sorge Gottes um den Menschen bezeichnet wird, darf man sie nach dem letzten Vertreter der platonischen Akademie nicht so vorstellen, als wendete sich Gott den Menschen zu, wie er sich auch nicht abwendet. Das geht aus einer Auseinandersetzung des Simplikios mit der christlichen Verzeihenslehre hervor, die an anderer Stelle entfaltet ist ${ }^{77}$. Für den Providenzbegriff ergibt sich aus dieser Auseinandersetzung, daß die göttliche Sorge-Providenz nicht eine Annäherung an die menschlichen Dinge beinhaltet. Wenn der Mensch sittliche Verfehlungen begeht, „ziehen wir uns selbst von dort weg“, ohne doch der alles durchdringenden göttlichen Providenz entrinnen zu können. Vielmehr verschaffen wir uns, wenn wir an der Krankheit der Schlechtigkeit leiden, am ehesten Zugang zur Heilung, die im Recht besteht, wenn wir uns der göttlichen Providenz und Sorge würdig machen, und genau das meint Platons berühmte Rede von der Homoiosis Theo. Wer dagegen diese unsere Hinwendung zu Gott als seine Hinwendung zu uns versteht - wie die Christen es tun - dem ergeht es so wie gewissen Schiffbrüchigen, die ein Seil um einen Felsen werfen, um sich und das Boot dem Felsen zu nähern und dabei der irrigen Meinung verfallen, daß nicht sie sich dem Felsen näherten, sondern der Felsen zu ihnen käme. In diesem Bild entsprechen die Reue, das Gebet und das Bittflehen dem Seil, denn durch sie vollzieht sich die „Hinwendung ${ }^{478}$. Hinwendung im Sinne der epistrophé

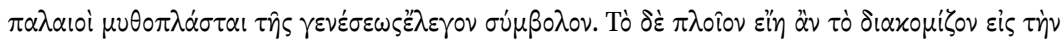

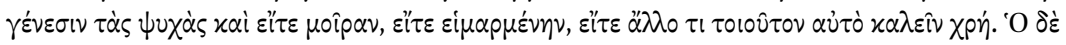

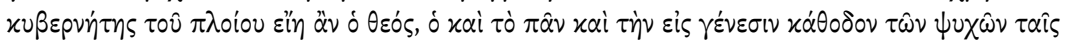

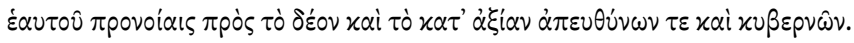

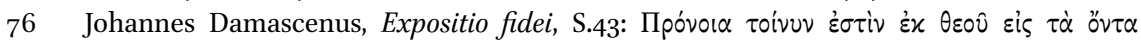

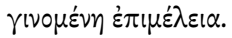

77 Cf. Th. Kobusch, Selbstwerdung und Personalität. Spätantike Philosophie und ihr Einfluß auf die Moderne (Tübingen: Mohr Siebeck, 2018), Kap. XVII.

78 Simplikios, Commentarius in Epicteti enchiridion, 36o,683-392,703. Das schöne Bild ist aufgenommen worden von Friedrich Heinrich Jacobi, Von den göttlichen Dingen, hg.v. W. Jaeschke (Hamburg: Meiner, 2000), 41,28-42,02: „So macht Simplicius, in seiner Auslegung des Epictet, wider gewisse Leute, die sich über die Zukehrung des Menschen zu Gott so ausdrückten, als wenn Gott, vom Menschen abgewendet, sich erst zu ihm wenden müßte, die Anmerkung: ,es ginge ihnen hiebey wie denen, die ein Schiffseil um einen Felsen am Ufer würfen, und da sie sich und ihr Boot an demselben Ufer hinan zögen, 
ist so - nach Simplikios - die Sache allein des Menschen bzw. des "Geistes“. Die göttliche Straf- oder Vergeltungsprovidenz ist nach dem nichtchristlichen Neuplatonismus keine Hinwendung zu den Menschen. Vielmehr ist für den paganen Philosophen der Gedanke einer Hinwendung Gottes zum Niedrigen skandalös. Die göttliche Hinwendung zum Menschen ist für den christlichen Philosophen ein Zeichen der Vollkommenheit Gottes, für den paganen Neuplatoniker ein Zeichen der Unvollkommenheit. Vor fast sechzig Jahren schrieb schon P. Aubin in diesem Sinne: „On sent très bien que la pensée philosophique de cette époque répugne à répudier systématiquement toute ,conversion' ad inferiora (...) mais nous n'avons pas trouvé d'auteur paien qui ose parler explicitement d'E- [i.e. ह̇ $\pi\llcorner\sigma \tau p \circ \varphi \eta ́$ ] de Dieu vers l'homme. (...) Tout E-

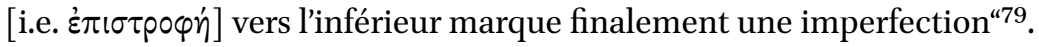

\section{$3 \quad$ Konklusion}

Die Lehre von der doppelten göttlichen Providenz, der providentia naturalis und der providentia voluntaria, die bei Berthold von Moosburg unter der Voraussetzung der Differenz von Philosophie und Theologie entwickelt wird, ist auf Dietrich von Freiberg zurückführbar. Die Unterscheidung zwischen dem Natürlichen und dem Willensmäßigen, die in den lateinischen Ausdrücken mit enthalten ist, stammt von Augustinus. Was bei diesen christlichen Denkern als Gründungsprovidenz und Erhaltungs- oder Rettungsprovidenz erscheint, das war im Neuplatonismus als demiurgische Providenz und Vergeltungsprovidenz (Dike, Heimarmene) vorgebildet worden.

\section{Bibliographie}

\section{Primäre Quellen}

Albert der Große, In IV Sententiarum, hg.v. A. Borgnet, Paris, Vivès, 1894.

Alexander von Hales, Summa universis theologiae, ed. Quaracchi, Roma, Coll. S. Bonaventurae, 1924-1948.

Ammonios, In Aristotelis Categorias commentaris, hg.v. A. Busse, Berlin, Reimer, 1895.

einfältig genug wären zu meinen, daß sie nicht sich dem Felsen näherten, sondern der Felsen allmählig zu ihnen käme“.

79 Cf. P. Aubin, Le problème de la conversion. Étude sur un terme commun à l'hellénisme et au christianisme des trois premiers siècles (Paris: Beauchesne, 1963), S.192-193. 
Ammonios, In Aristotelis librum De interpretatione commentarius, hg.v. A. Busse, Berlin, Reimer, 1897.

Augustinus, De Genesi ad litteram, hg.v. J. Zycha, Prag / Leipzig / Wien, Tempsky / Freytag, 1894 .

Augustinus, De opere monachorum, hg.v. J. Zycha, Wien, Tempsky, 1900.

Augustinus, De vera religione, hg.v. J. Clémence, J. Pegon, Paris, Desclée de Brouwer, 1951.

Augustinus, Enarrationes in Psalmos, hg.v. E. Deckers, I. Fraipont, Turnhout, Brepols, 1956.

Augustinus, Contra Secundinum, hg.v. R. Jolivet, M. Jourjon, Paris, Desclée de Brouwer, 1961.

Augustinus, De catechizandis rudibus, hg.v. I.B. Bauer, Turnhout, Brepols, 1969.

Augustinus, Epistulae, hg.v. K.D. Daur, Turnhout, Brepols, 2009.

Augustinus, De civitate Dei, hg.v. B. Dombart, A. Kalb, G. Bardy, G. Combès, Paris, Institut d'études augustiniennes, 2014.

Berthold von Moosburg, Expositio super Elementationem theologicam Procli. Propositiones 1-13, hg.v. M.R. Pagnoni-Sturlese, L. Sturlese, Hamburg, Meiner, 1984.

Berthold von Moosburg, Expositio super Elementationem theologicam Procli. Propositiones 14-34, hg.v. L. Sturlese, M.R. Pagnoni-Sturlese, B. Mojsisch, Hamburg, Meiner, 1986.

Berthold von Moosburg, Expositio super Elementationem theologicam Procli. Propositiones 35-65, hg.v. A. Sannino, Hamburg, Meiner, 2001.

Berthold von Moosburg, Expositio super Elementationem theologicam Procli. Propositiones 66-107, hg.v. I. Zavattero, Hamburg, Meiner, 2003.

Berthold von Moosburg, Expositio super Elementationem theologicam Procli. Propositiones 108-135, hg.v. F. Retucci, Hamburg, Meiner, 2011.

Berthold von Moosburg, Expositio super Elementationem theologicam Procli. Propositiones 136-159, hg.v. F. Retucci, Hamburg, Meiner, 2007.

Berthold von Moosburg, Expositio super Elementationem theologicam Procli. Propositiones 16o-183, hg.v. U.R. Jeck, I.J. Tautz, Hamburg, Meiner, 2003.

Berthold von Moosburg, Expositio super Elementationem theologicam Procli. Propositiones 184-211, hg.v. L. Sturlese, Hamburg, Meiner, 2014.

Dietrich von Freiberg, Opera omnia, vol. ı. Schriften zur Intellekttheorie, ed. B. Mojsisch, Hamburg, Meiner, 1977.

Dietrich von Freiberg, Opera omnia, vol. 2. Schriften zur Metaphysik und Theologie, hg.v. R. Imbach et al., Hamburg, Meiner, 198o.

Dietrich of Freiberg, Opera omnia, vol. 3. Schriften zur Naturphilosophie und Metaphysik, hg.v. J.-D. Cavigioli et al., Hamburg, Meiner, 1983.

Dietrich von Freiberg, Opera omnia, vol. 4. Schriften zur Naturwissenschaft, Briefe, hg.v. M.R. Pagnoni-Sturlese et al., Hamburg, Meiner, 1985. 
Heinrich von Gent, Summa (Quaestiones ordinariae). Art. $X X X V-X L$, hg.v. G.A. Wilson, Leuven, Leuven University Press, 1994.

Heinrich von Gent, Summa (Quaestiones ordinariae). Art. I-IV, hg.v. G.A. Wilson, Leuven, Leuven University Press, 2005.

Heinrich von Gent, Summa (Quaestiones ordinariae). Art. XLVII-LII, hg.v. M. Führer, Leuven, Leuven University Press, 2008.

Hermias, In Platonis Phaedrum Scholia, hg.v. C.M. Lucarini, C. Moreschini, Berlin, De Gruyter, 2012.

Hierokles, Peri Pronoias v. Photius, Bibliotheka.

Hierokles, In Aureum Pythagoreorum Carmen Commentarius, hg.v. F.W. Koehler, Stuttgart, Teubner, 1974.

Jacobi, F.H., Von den göttlichen Dingen, hg.v. W. Jaeschke, Hamburg, Meiner, 2000.

Jamblich, Theologumena arithmeticae hg.v. V. de Falco, Leipzig, Teubner, 1922.

Jamblich, De mysteriis, hg.v. E. des Places, Paris, Les Belles Lettres, 1989.

Johannes Damascenus, Expositio fidei, hg.v. P.B. Kotter, Berlin / New York, De Gruyter, 1973.

Johannes Philoponos, In Aristotelis De anima libros, hg.v. M. Hayduck, Berlin, Reimer, 1897.

Johannes Philoponos, In Aristotelis Categorias commentarius, hg.v. A. Busse, Berlin, Reimer, 1898.

Johannes Philoponos, De aeternitate mundi contra Proclum, hg.v. H. Rabe, Leipzig, Teubner, 1899 (repr. 1963).

Johannes Philoponos, In Aristotelis Analytica priora commentaria, hg.v. M. Wallies, Berlin, Reimer, 1905 .

Nikolaus von Straßburg, Summa, liber II, tract. 8-14, hg.v. T. Suarez-Nani, Hamburg, Meiner, 1990.

Olympiodor, In Platonis Gorgiam, hg.v. L.G. Westerink, Leipzig, Teubner, 1970.

Olympiodor, In Platonis Phaedonem Commentaria, hg.v. L.G. Westerink, Amsterdam, North-Holland, 1976.

Photius, Bibliothèque, hg.v. R. Henry, Paris, Les Belles Lettres, 1959-1991.

Porphyrios, In Platonis Timaeum Commentariorum Fragmenta, hg.v. A.R. Sodano, Neapel, Istituto della Stampa, 1964.

Porphyrios, Ad Marcellam, hg.v. A. Nauck und übers. von W. Pötscher, Leiden, Brill, 1969.

Proclus, In Platonis Rempublicam Commentaria, hg.v. W. Kroll, Leipzig, Teubner, 1901 (repr. Amsterdam, Hakkert, 1965).

Proclus, In Platonis Timaeum commentaria, hg.v. E. Diehl, Leipzig, Teubner, 1903 (repr. Amsterdam, Hakkert, 1965).

Proclus, Théologie platonicienne, lib. I, hg.v. H.D. Saffrey, L.G. Westerink, Paris, Les Belles Lettres, 1968. 
Proclus, Théologie platonicienne, lib. v, hg.v. H.D. Saffrey, L.G. Westerink, Paris, Les Belles Lettres, 1987 .

Proclus, Théologie platonicienne, lib. vi, hg.v. H.D. Saffrey, L.G. Westerink, Paris, Les Belles Lettres, 1997.

Proclus, Sur le Premier Alcibiade de Platon, I, hg.v. A.-Ph. Segonds, Paris, Les Belles Lettres, 2003.

Proclus, De decem dubitationibus circa providentiam, hg.v. H. Boese, Berlin, De Gruyter, 1960.

Proclus, Elementatio theologica, hg.v. E.R. Dodds, Oxford, Clarendon Press, $1963^{2}$.

Proclus, In Platonis Parmenidem Commentaria, hg.v. C. Steel, Oxford, Oxford University Press, 2008.

Simplikios, In Aristotelis Physicorum libros commentaria, hg.v. H. Diels, Berlin, Reimer, 1882.

Simplikios, Commentarius in Epicteti enchiridion, hg.v. I. Hadot, Leiden / New York, Brill, 1996.

Thomas von Aquin, Summa contra Gentiles, hg.v. P. Marc, C. Pera, P. Caramello, Torino / Roma, Marietti, 1961.

Thomas von Aquin, Summa Theologiae, Prima pars, hg. Leonina, Roma, Ex Typ. Polyglotta S.C. de Propaganda Fide, 1888-1889.

\section{Sekundäre Quellen}

Aubin, P., Le problème de la conversion. Étude sur un terme commun à l'hellénisme et au christianisme des trois premiers siècles, Paris, Beauchesne, 1963.

Flasch, K., „Einleitung“, in Dietrich von Freiberg, Opera omnia, vol. 1. Schriften zur Intellekttheorie, S.IX-XXVI.

Flasch, K., "Einleitung", in Berthold of Moosburg, Expositio super Elementationem theologicam Procli. Prologus, Propositiones 1-13, p. xi-Xxxviii.

Führer, M., „Dietrich of Freiberg“, in E.N. Zalta (Hg.), The Stanford Encyclopedia of Philosophy (Fall 2020 Edition), URL = https://plato.stanford.edu/archives/fall2O2O/ entries/dietrich-freiberg/ (29.11.2020).

Goris, W., „Das Gute als Ersterkanntes bei Berthold von Moosburg“, in W. Goris (Hg.), Die Metaphysik und das Gute. Aufsätze zu ihrem Verhältnis in Antike und Mittelalter. Jan A. Aertsen zu Ehren, Leuven, Peeters, 1999, S.143-145.

Kobusch, Th., „Begriff und Sache. Die Funktion des menschlichen Intellekts in der mittelalterlichen Philosophie“, in Internationale Zeitschrift für Philosophie 13/2(2004), S.140-157.

Kobusch, Th., „Zustimmung I. Antike; Mittelalter“, in J. Ritter, K. Gründer, G. Gabriel (Hgg.), Historisches Wörterbuch der Philosophie, Basel / Stuttgart, Schwabe AG, 2005, S.1457-1465. 
Kobusch, Th., Christliche Philosophie. Die Entdeckung der Subjektivität, Darmstadt, Wissenschaft Buchgesellschaft, 2006.

Kobusch, Th., „Der Begriff des Willens in der christlichen Philosophie vor Augustinus“, in J. Müller, R. Hofmeister Pich (Hgg.), Wille und Handlung in der Philosophie der Kaiserzeit und Spätantike. Will and Action in Late Antique Philosophy, Berlin / New York, De Gruyter, 2010, S.277-30o.

Kobusch, Th., „Die Vernunftordnung als Vollendung der Naturordnung. Zur Funktion der menschlichen Vernunft in der mittelalterlichen Philosophie“, in T. Iremadze (mit H. Schneider, K.J. Schmidt) (Hgg.), Philosophy in Global Change: Jubilee volume dedicated to the 65th anniversary of Burkhard Mojsisch, Tbilisi, Nekeri, 2011, S.140-154.

Kobusch, Th., Die Philosophie des Hoch- und Spätmittelalters, München, Beck, 2011.

Kobusch, Th., „Origenes und Pico. Picos Oratio im Licht der spätantiken Philosophie“, in A. Fürst, Chr. Hengstermann (Hgg.), Origenes Humanista. Pico della Mirandolas Traktat De salute Origenis disputatio, Münster, Aschendorff, 2015, S.141-159.

Kobusch, Th., Selbstwerdung und Personalität. Spätantike Philosophie und ihr Einfluß auf die Moderne, Tübingen, Mohr Siebeck, 2018.

Kobusch, Th., "Complicatio und explicatio. Das ontologische Modell des Neuplatonismus“, in G, Kapriev (hg.), The Dionysian Traditions, Turnhout, Brepols, 2021.

Retucci, F., „Magister Thomas Anglicus Minor. Eine neue Quelle der Expositio super Elementationem theologicam Procli Bertholds von Moosburg - das ungedruckte Sapientiale des Franziskaners Thomas von York“, in Berthold of Moosburg, Expositio - Prop. 136-159, S.XXIII-XXXIX.

Theiler, W., „Ammonios der Lehrer des Origenes“, in Id., Forschungen zum Neuplatonismus, Berlin, De Gruyter, 1966, S.1-45. 